\title{
Effects of Exercise Programs on Physical Factors and Safety in Adult Patients with Cancer and Haematopoietic Stem Cell Transplantation: A Systematic Review
}

\author{
Erica Morales-Rodriguez ${ }^{1,2} \mathbb{D}$, Txomin Pérez-Bilbao ${ }^{2,3}$, Alejandro F. San Juan ${ }^{2, *},+\mathbb{D}$ and Jorge Lorenzo Calvo ${ }^{1,+} \mathbb{D}$ \\ 1 Sports Department, Faculty of Physical Activity and Sports Sciences-INEF, Universidad Politécnica de \\ Madrid, 28040 Madrid, Spain; em.morales@alumnos.upm.es (E.M.-R.); jorge.lorenzo@upm.es (J.L.C.) \\ 2 Department of Health and Human Performance, Faculty of Physical Activity and Sports Sciences-INEF, \\ Universidad Politécnica de Madrid, 28040 Madrid, Spain; tperez@comillas.edu \\ 3 Department of Education, Investigation Methods and Evaluation, Faculty of Human and Social Sciences, \\ Universidad Pontificia de Comillas, 28049 Madrid, Spain \\ * Correspondence: alejandro.sanjuan@upm.es \\ + Lorenzo Calvo, J. and San Juan, A.F. share senior authorship.
}

Citation: Morales-Rodriguez, E.; Pérez-Bilbao, T.; San Juan, A.F.; Calvo, J.L. Effects of Exercise Programs on Physical Factors and Safety in Adult Patients with Cancer and Haematopoietic Stem Cell Transplantation: A Systematic Review. Int. J. Environ. Res. Public Health 2022, 19, 1288. https:// doi.org/10.3390/ijerph19031288

Academic Editor: Paul B.

Tchounwou

Received: 5 December 2021

Accepted: 17 January 2022

Published: 24 January 2022

Publisher's Note: MDPI stays neutral with regard to jurisdictional claims in published maps and institutional affiliations.

Copyright: (c) 2022 by the authors. Licensee MDPI, Basel, Switzerland. This article is an open access article distributed under the terms and conditions of the Creative Commons Attribution (CC BY) license (https:// creativecommons.org/licenses/by/ $4.0 /)$.

\begin{abstract}
This study looks at the effects of exercise programs on physical factors and safety in adult patients with cancer and hematopoietic stem cell transplantation (HSCT) or bone marrow transplantation (BMT). A systematic search was performed in the PubMed and Web of Science databases (from inception to 26 August 2021). A review was carried out following the Preferred Reporting Items for Systematic review and Meta-Analyses (PRISMA) checklist. The methodological quality of the included studies was assessed with the Physiotherapy Evidence Database (PEDro) scale, based, in turn, on the Delphi list. A total of 25 randomized controlled trials studies were included, comprising 1434 patients. The most significant result of this systematic review is that exercise program interventions are safe and produce positive changes in cardiorespiratory fitness, muscle strength, and the functional mobility-state in adult patients with cancer and HSCT or BMT. Only 3 patients from the 711 participants in the exercise interventions (i.e., $0.42 \%$ ) reported adverse events related to exercise interventions. Moreover, exercise training programs may have a cardiological and muscular protective effect, as well as a healthy effect on the prevention and control of transplant complications, improving health outcomes.
\end{abstract}

Keywords: cancer; hematopoietic stem cell transplantation; bone marrow transplant; exercise; fitness

\section{Introduction}

Bone marrow transplantation (BMT), or hematopoietic stem cell transplantation (HSCT), is a treatment for hematological pathologies [1] such as myelosuppression produced by high dose chemotherapy (HDC), neoplastic syndromes, immunodeficiency, and enzyme deficiencies [2]. Autologous HSCT (i.e., stem cells come from the patient's own blood or bone marrow) is the most common; it depends on the conditioning therapy that precedes transplantation and on hematopoietic growth factors that can reduce cytopenia after transplantation [3]. Allogeneic HSCT (Allo-HSCT), involving genetically similar donors, is associated with the side effects of treatment or graft versus host disease (GVHD) in $50 \%$ of its recipients [4]. The 5-year survival rate is $80 \%$ [5,6], with a $59 \%$ cumulative incidence of developing a disease [7].

Antineoplastic treatments have side effects [3] such as: the loss of physical performance [8], fatigue $[9,10]$, and a weak immune and hematological system that sometimes lead to HSCT [11]. HSCT is related to the appearance of: pain, dizziness, asthenia, anemia, cytopenia, nausea, vomiting, diarrhea, infections, and fever related to aplasia and length of hospitalization [12]. Functional capacity is directly related to physical capacity as an 
indicator of health in pathological processes [13], and they serve for medical diagnosis [14]. Overall, $25 \%$ of cancer patients suffer a functional decline due to a decrease in physical activity, such as: loss of cardiovascular function, muscle strength, and lung function, as well as a reduction in lean body tissue [15].

In the past, physicians recommended patients with chronic diseases (e.g., cardiovascular diseases, pulmonary disorders, cancer) to avoid physical activity. However, in 1960s, numerous clinical studies showed the benefits of exercise on cardiac rehabilitation. These studies, together with the recommendations of the World Health Organization (1964), pushed the establishment and development of cardiac rehabilitation based on exercise [16]. In oncologic patients, the first report about the benefits of exercise was in 1980s, showing mood-enhancing effects during antineoplastic treatment [17]. In 1989, the first randomized controlled trial in 45 women with breast cancer under chemotherapy was realized. After 10 weeks of an aerobic exercise intervention, they observed a significant improvement in the maximum oxygen consumption $\left(\mathrm{VO}_{2 \max }\right)$ [18]. From then on, the scientific evidence of exercise programs increased exponentially and pushed the establishment of oncologic rehabilitation based on exercise $[19,20]$.

Specifically, the first study focusing on the functional capacity recovery of cancer patients after BMT was carried out by Dimeo et al. [21]. They developed an aerobic exercise program for 6 weeks and observed significant improvements in maximum physical performance and walking distance and a diminished heart rate with equivalent workloads [21]. It is currently known that exercise programs in patients with HSCT-BMT produce improvements in cardiorespiratory fitness (CRF), which is a predictor of mortality and cardiovascular diseases [22], the main indicator of which is the maximum oxygen consumption $\left(\mathrm{VO}_{2 \max }\right)$. They also enhance muscle strength $[1,23,24]$, metabolic and immune function [25], reduce the side effects of cancer treatment [26], and influence creatine, haemoglobin concentration, and hospitalization time [27,28]. However, there are limitations with existing studies, such as the joint inclusion of allogeneic and autologous patients [21,29], few control groups [30], or a small sample size [31].

The aim of this systematic review is to provide an updated analysis of data from experimental studies that have examined the effects of exercise programs on physical factors and safety in adult patients with cancer and HSCT or BMT.

\section{Materials and Methods}

\subsection{Search Strategy}

We used the Preferred Reporting Items for Systematic Reviews and Meta-analysis (PRISMA) to conduct this systematic review, as well as its possible consequences for the risk of bias [32]. An electronic search was realized for articles written in English in the electronic databases PubMed and Web of Science (from inception until 26 August 2021). We used the Mesh-indexed terms from PubMed and the following search strategy: (bone marrow transplant OR hematopoietic stem cell transplantation) AND (exercise OR physical activity) AND (immune system OR cardiovascular function OR neuromuscular function OR oxygen consumption OR strength OR resistance training OR acceleration OR mobility OR range of motion OR health OR neoplasms OR cancer survivors).

\subsection{Selection of the Studies}

Published pilot studies were included. Unpublished clinical trials registered in clinicaltrials.gov that provided results, grey literature (e.g., abstracts, conference proceedings, and editorials), and reviews were excluded. A difference was made between exercise programs pre- and post-HSCT in cancer patients. All the selected studies met the following inclusion criteria: (a) published in English; (b) randomized controlled trials (RCTs); (c) adult patients (age $\geq 18$ years old) of both sexes who suffered or had suffered from any type of cancer at the time of the study; (d) patients in the process of receiving or who had received an HSCT; and (e) patients who had undergone an exercise program intervention. The studies exclusion criteria were: (a) participants under 18 years of age; (b) participants were not 
humans; (c) patients not related to cancer; (d) studies that did not include an exercise program; (e) studies related with other diseases or themes. The selection of the studies was realized by two authors independently (E.M.R and J.L.C.), and disagreements were resolved through discussion with a third author (T.P.B.).

\subsection{Data Extraction}

Two authors (E.M.R. and J.L.C.) independently extracted the following data from each study: sample (N); sex and age of participants; type of cancer; characteristics of the interventions (type, equipment, frequency, intensity, duration, session, rest, supervision, adjustment); relevant clinical variables and significant results. If there were disagreements in data extraction, authors discussed until a consensus was reached.

\subsection{Risk of Bias Assessment}

Two authors (E.M.R and J.L.C.) independently scored the studies, applying the Physiotherapy Evidence Database (PEDro) scale, based, in turn, on the Delphi list [33], and disagreements were resolved through discussion with a third author (T.P.B.). The total score of the PEDro scale was from 0 to 10, counting the number of criteria met by each study (see footnotes in Table 1). The quality of the study was rated as poor (PEDro score $\leq 3$ ), fair (4-5), or high ( $\geq 6)$.

Table 1. Quality of the studies included in the systematic review.

\begin{tabular}{|c|c|c|c|c|c|c|c|c|c|c|c|c|}
\hline \multicolumn{13}{|c|}{ Items } \\
\hline Study & 1 & 2 & 3 & 4 & 5 & 6 & 7 & 8 & 9 & 10 & 11 & Total Score \\
\hline (Baumann et al., 2010) [23] & + & + & + & + & - & - & - & + & + & + & + & 7 \\
\hline (Jarden et al., 2007) [31] & + & + & + & + & + & $?$ & $?$ & + & + & + & + & 9 \\
\hline (Shelton et al., 2009) [34] & + & + & + & + & - & - & - & + & + & + & + & 7 \\
\hline (DeFor et al., 2007) [30] & + & + & + & $?$ & $?$ & $?$ & $?$ & + & + & + & + & 6 \\
\hline (Barğ1 et al., 2016) [35] & + & + & + & + & + & + & - & + & + & + & + & 9 \\
\hline (Wiskemann et al., 2011) [36] & + & + & + & + & $?$ & $?$ & ? & + & + & + & + & 7 \\
\hline (Hacker et al., 2011) [37] & + & + & + & + & $?$ & $?$ & $?$ & + & + & + & + & 7 \\
\hline (Baumann et al., 2011) [38] & + & + & + & + & - & - & - & + & + & + & + & 7 \\
\hline (Coleman et al., 2003) [39] & + & + & + & + & $?$ & $?$ & $?$ & + & + & + & + & 7 \\
\hline (Jarden et al., 2009) [40] & + & + & + & + & + & - & - & + & + & + & + & 8 \\
\hline (KIM \& KIM, 2006) [41] & + & + & + & + & - & - & - & + & + & + & + & 7 \\
\hline (Jarden et al., 2009) [42] & + & + & + & + & - & - & - & + & + & + & + & 7 \\
\hline (Knols et al., 2011) [43] & + & + & + & + & + & - & + & + & + & + & + & 10 \\
\hline (Schumacher et al., 2018) [44] & + & + & + & + & + & $?$ & $?$ & + & + & + & + & 8 \\
\hline (Wiskemann et al., 2014) [45] & + & + & + & + & + & - & - & + & + & + & + & 8 \\
\hline (Mello et al., 2003) [46] & + & + & $?$ & + & $?$ & $?$ & $?$ & + & + & + & + & 6 \\
\hline (Wiskemann et al., 2015) [47] & + & + & $?$ & + & $?$ & $?$ & $?$ & + & + & + & + & 6 \\
\hline (Peters et al., 2018) [48] & + & + & + & + & + & - & - & + & + & + & + & 8 \\
\hline (Pahl et al., 2018) [49] & + & + & + & + & - & - & - & + & + & + & + & 7 \\
\hline (Persoon et al., 2017) [50] & + & + & + & + & + & + & + & + & + & + & + & 10 \\
\hline (Bird et al., 2010) [51] & + & + & + & + & - & - & - & + & + & + & + & 7 \\
\hline (Oechsle et al., 2014) [52] & + & + & - & + & - & - & - & + & + & + & + & 6 \\
\hline (Van Dongen et al., 2019) [53] & + & + & + & + & - & - & - & + & + & + & + & 7 \\
\hline
\end{tabular}


Table 1. Cont.

\begin{tabular}{|c|c|c|c|c|c|c|c|c|c|c|c|c|}
\hline \multicolumn{13}{|c|}{ Items } \\
\hline Study & 1 & 2 & 3 & 4 & 5 & 6 & 7 & 8 & 9 & 10 & 11 & Total Score \\
\hline (Pahl et al., 2020) [54] & + & + & + & + & + & - & - & + & + & + & + & 8 \\
\hline (Almeida et al., 2020) [55] & + & + & + & + & + & - & - & + & + & + & + & 8 \\
\hline
\end{tabular}

Column numbers correspond to the following criteria on the PEDro scale: 1-Eligibility criteria were specified. 2-Subjects were randomly allocated to groups (or, in a crossover study, subjects were randomly allocated an order in which treatments were received). 3-Allocation was concealed. 4-Groups were similar at baseline. 5-Subjects were blinded. 6-Therapists who administered the treatment were blinded. 7-Assessors were blinded. 8-Measures of key outcomes were obtained from more than $85 \%$ of subjects. 9-Data were analyzed by intention to treat. 10-Statistical comparisons between groups were conducted. 11-Point measures and measures of variability were provided. A total score out of 10 is determined from the number of criteria that are satisfied, except that scale item 1 is not used to generate the total score. + Indicates the criterion was clearly satisfied; indicates that it was not; ? indicates that it is not clear whether the criterion was satisfied.

\section{Results}

\subsection{Study Selection}

A total of 10,467 references were identified in the database. After eliminating duplicate studies $(n=9409), 1058$ studies remained. After selection by title, abstract, and full text, 1033 articles were excluded, and 25 studies met the inclusion criteria (Figure 1).

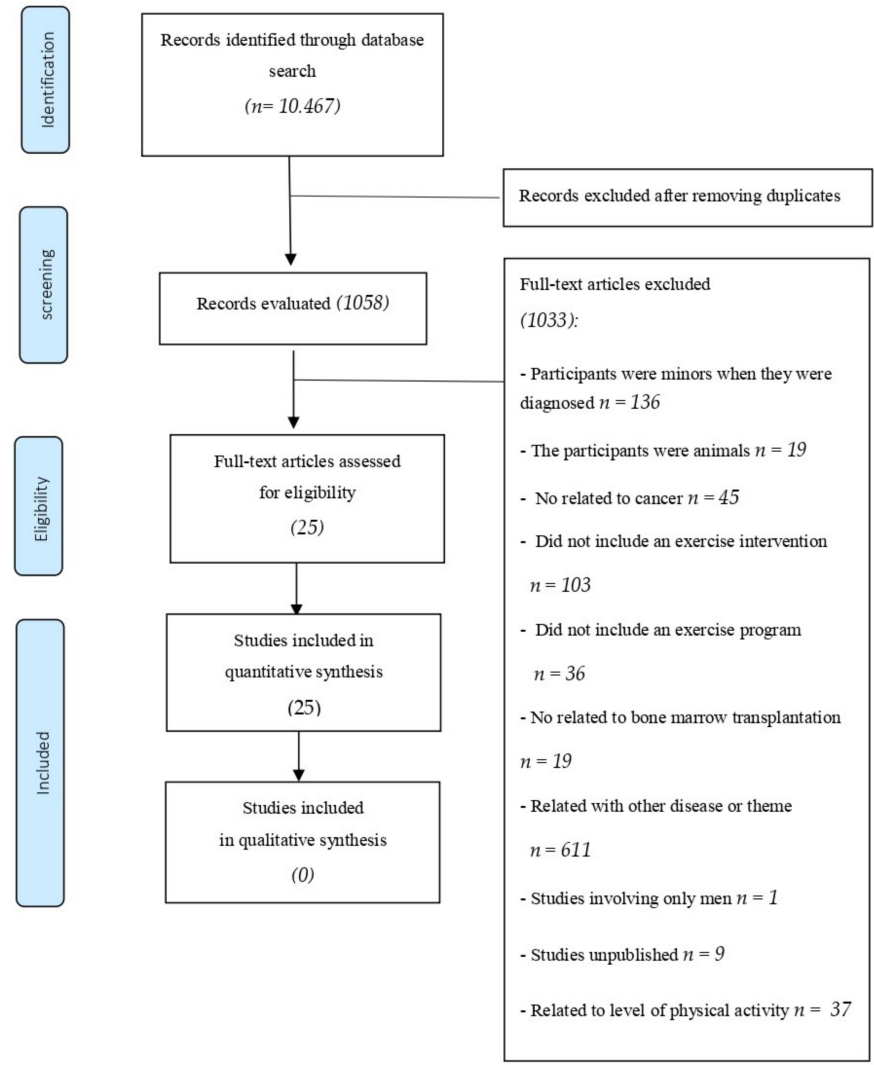

Figure 1. Systematic review flow chart.

\subsection{Study Characteristics}

Twenty-five studies were included and analyzed in this systematic review [23,30,31,34-55] (Table 1). The characteristics of the included studies are summarized in Tables 2 and 3. All studies included were RCTs with a control group (CT), with no exercise intervention, and an intervention group (EXP) except the study [34], which divided the sample size into a supervised or self-directed exercise program. 
Table 2. Studies that have analyzed the effects of an exercise program before and after HSCT, with main results in physically related variables.

\begin{tabular}{|c|c|c|c|c|c|}
\hline Study & Study Design & $\begin{array}{l}\text { Sample Size by Group (Sex), Age } \\
\text { (Mean } \pm \text { SD; Range) }\end{array}$ & Primary Cancer & Intervention & $\begin{array}{l}\text { Main Results in Physical } \\
\text { Related Variables }\end{array}$ \\
\hline $\begin{array}{l}\text { (DeFor et al., } \\
\text { 2007) [30] }\end{array}$ & $\begin{array}{l}\text { RCT } \\
\text { T1: Pre HSCT (A) } \\
\text { T2: Post HSCT (100 days) }\end{array}$ & $\begin{array}{l}\text { - EXP: } n=51 \text { ( } 22 \text { female), } 46 \text { years } \\
(18-68) \\
\text { - CT: } n=49 \text { (17 female), } 49 \text { years } \\
(22-64)\end{array}$ & $\begin{array}{l}\text { AA, ALL, AML, CML, HL, } \\
\text { LHN, MDS }\end{array}$ & $\begin{array}{l}\text { Type: AT (walking on treadmill) } \\
\text { Duration: } 100 \text { days } \\
\text { Intensity: Comfortable speed } \\
\text { Supervised: not } \\
\text { Frequency: } 7 \text { times/week } \\
\text { Setting: Clinic/home }\end{array}$ & $\begin{array}{l}\text { T1-T2: } \\
-\downarrow \text { KPS: } \downarrow \text { EXP }(-10 \text { pts } / 100 \\
\text { pts) } \downarrow \text { CT (-20 pts } / 100 \text { pts }) \\
-\leftrightarrow \text { Immune system }\end{array}$ \\
\hline $\begin{array}{l}\text { (Wiskemann et al., } \\
\text { 2011) [36] }\end{array}$ & $\begin{array}{l}\text { RCT } \\
\text { T1: Pre HSCT (medical } \\
\text { checkup)-Pre HSCT ( } 4 \text { week } \\
\text { A) } \\
\text { T2: Post HSCT (H)-Post } \\
\text { HSCT (D) } \\
\text { T3: Post HSCT (D)-Post } \\
\text { HSCT (D 6-8 weeks) }\end{array}$ & $\begin{array}{l}\text { - EXP: } n=52 \text { (21 female), } 47.6 \text { years } \\
(18-70) \\
- \text { CT: } n=53,(13 \text { female), } 50 \text { years } \\
(20-71)\end{array}$ & $\begin{array}{l}\text { AA, ALL, AML, CLL, } \\
\text { CML, MDS, MM, MPS, } \\
\text { Others }\end{array}$ & $\begin{array}{l}\text { Type: AT (walking, stationary bicycle), ST (elastic bands) } \\
\text { Duration: } 18 \text { weeks } \\
\text { Intensity and volume: AT 1-4 week to (A) } 3 \text { times a week, } \\
\text { from (H) } 3-5 \text { times a week RPE (12-14/20), DCT: color codes } \\
\text { (Red 15-20 min, yellow } 20-30 \text { min, green } 30-40 \text { min), from } \\
1-8 \text { week rehabilitation } 3 \text { times a week. ST } 1-4 \text { week to (A) } \\
\text { twice a week, from (H) twice a week (2-3 sets of } 8-20 \text { reps } \\
\text { RPE } 14-16 / 20) \text {, from 1-8 week rehabilitation twice a week. } \\
\text { Supervised: yes T2 and self-directed T1 and T3 } \\
\text { Setting: Home/Hospital }\end{array}$ & $\begin{array}{l}\text { T1 } \\
-\uparrow 6 \mathrm{MWT} \\
(\Delta-0.19 \%) \\
-\leftrightarrow \text { Immune system }\end{array}$ \\
\hline $\begin{array}{l}\text { (Baumann et al., } \\
\text { 2011) [38] }\end{array}$ & $\begin{array}{l}\text { RCT } \\
\text { T1:Pre HSCT (A) } \\
\text { T2:Post HSCT (7-8 weeks) }\end{array}$ & $\begin{array}{l}\text { - EXP: } n=17,(6 \text { female }) \\
41.41 \pm 11.78^{\text {a }} \text { years } \\
-\mathrm{CT}=n=16,(11 \text { female }) \\
42.81 \pm 14.04 \text { a years }\end{array}$ & $\begin{array}{l}\text { ALL, AML, CLL, CML, } \\
\text { MDS, MM, MPS, PID }\end{array}$ & $\begin{array}{l}\text { Type: AT (stationary bicycle), ADL, stretching, coordination } \\
\text { Duration: } 7-8 \text { weeks } \\
\text { Volume and intensity: AT (H) once-twice a week (10-20 } \\
\text { min/day continuous or interval training at HRmax } 80 \%) \text {; } \\
\text { ADL-training (H) } 5 \text { times a day ( } 20 \text { min a day, } 5 \times 20 \text { steps } \\
\text { with } 1 \text { min break of slightly strenuous or strenuous); } \\
\text { mobilization passive and active } 1 \text { day after HSCT until } 1 \text { day } \\
\text { before hospital discharge daily except on weekends Low } \\
\text { intensity; or not strenuous (CT) } 20 \text { min a day } \\
\text { Cadence: AT cycle (since } 25 \mathrm{~W} \text { with } 25 \mathrm{~W} \text { increment every } \\
2 \text { min) } \\
\text { Supervised: yes } \\
\text { Setting: hospital }\end{array}$ & $\begin{array}{l}\text { T1-T2: } \\
\text { - Endurance: } \downarrow \text { CT } \\
\text { ( } \Delta \text {-23.5\%) } \\
\text { - Relative endurance: } \downarrow \text { CT } / \uparrow \\
\text { EXP } \\
(\Delta-15.8 \% ; \Delta 11.02 \%) \\
\text { - Strength lower extremities: } \\
\downarrow \text { CT } \\
(\Delta-26.8 \%) \\
\text { - BMI: } \downarrow \text { CT } / \downarrow \text { EXP } \\
(\Delta-8.2 \% ; \Delta-13.2 \%) \\
-\leftrightarrow \text { Immune system }\end{array}$ \\
\hline $\begin{array}{l}\text { (Coleman et al., } \\
\text { 2003) [39] }\end{array}$ & $\begin{array}{l}\text { RCT with RM } \\
\text { T1:Pre HSCT (A) } \\
\text { T2: Post HSCT (3 months) }\end{array}$ & $\begin{array}{l}\text { - EXP: } n=14 \\
\text { - CT: } n=10 ;(10 \text { female), } 55 \text { years } \\
\text { (42-74) }\end{array}$ & $\mathrm{MM}$ & $\begin{array}{l}\text { Type: AT (walking), ST (elastic bands) } \\
\text { Duration: } 6 \text { months } \\
\text { Intensity and volume: AT } 3 \text { times a week ( } 18 \text { min fast-paced } \\
\text { walking at RPE } 12-15 / 20) \text {, ST } 3 \text { times a week with color } \\
\text { bands ( } 1 \text { set of } 8 \text { red } 9-15 \mathrm{Ib}, 1 \text { set of } 8 \text { green } 5-9 \mathrm{Ib} \text { ) and ( } 2 \\
\text { sets of } 8 \text { chair stands of } 1 \mathrm{RM}) \\
\text { Supervised: not } \\
\text { Setting: home }\end{array}$ & $\begin{array}{l}\text { T1-T2: } \\
\text { - LBW: } \uparrow \text { EXP } / \downarrow \text { CT } \\
(\Delta 0.1 \% ; \Delta-3.6 \%) \\
\text { - Strenght: } \uparrow \text { EXP } / \downarrow C T \\
(\Delta 2.4 \% ; \Delta-12.6 \%) \\
-\leftrightarrow \text { Immune system }\end{array}$ \\
\hline
\end{tabular}


Table 2. Cont.

\begin{tabular}{|c|c|c|c|c|c|}
\hline Study & Study Design & $\begin{array}{l}\text { Sample Size by Group (Sex), Age } \\
\text { (Mean } \pm \text { SD; Range) }\end{array}$ & Primary Cancer & Intervention & $\begin{array}{l}\text { Main Results in Physical } \\
\text { Related Variables }\end{array}$ \\
\hline $\begin{array}{l}\text { (Jarden et al., } \\
\text { 2009) [42] }\end{array}$ & $\begin{array}{l}\text { RCT } \\
\text { T1: Pre HSCT (A) } \\
\text { T2: Post HSCT ( } 6 \text { weeks) }\end{array}$ & $\begin{array}{l}\text { - EXP: } n=21 \text {, (8 female), } 40.9 \text { years } \\
(18-60) \\
\text { - CT: } n=21 \text {, (8 female), } 37.4 \text { years } \\
(18-55)\end{array}$ & $\begin{array}{l}\text { AA, ALL, AML, CML, } \\
\text { MDS, MF, PNH, WM }\end{array}$ & $\begin{array}{l}\text { Type: AT (stationary bicycle), ST (hand and ankle weights), } \\
\text { stretching, relaxation } \\
\text { Duration: } 4-6 \text { weeks } \\
\text { Intensity and volume: AT } 5 \text { times a week (HRmax } 50-75 \% \\
\text { Low to moderate RPE } 10 / 13 \text { ), stretching (Dynamic: } 1-2 \text { sets } \\
\text { of } 10-12 \text { reps; Static: } 1 \text { set/15-30 seg), ST } 3 \text { times a week } \\
\text { (1-2 sets of } 10-12 \text { reps at low to moderate, RPE 10/13) and } \\
\text { relaxation twice a week (Low RPE 6/9) } \\
\text { Cadence: } 30-70 \text { cycles / min and range at 30-75 W. } \\
\text { Supervised: yes } \\
\text { Setting: Hospital }\end{array}$ & $\begin{array}{l}\text { T1-T2: } \\
-\leftrightarrow \text { Immune system }\end{array}$ \\
\hline $\begin{array}{l}\text { (Jarden et al., } \\
\text { 2009) [40] }\end{array}$ & $\begin{array}{l}\text { RCT } \\
\text { T1: Pre HSCT (A) } \\
\text { T2: PostHSCT (D) }\end{array}$ & $\begin{array}{l}\text { - EXP: } n=21 \text { ( } 8 \text { female), } 45.0 \text { years } \\
(18-60) \\
- \text { CT: } n=21 \text { ( } 8 \text { female), } 38.0 \text { years } \\
(18-55)\end{array}$ & $\begin{array}{l}\text { AA, ALL, AML, CML, } \\
\text { MDS, MF, PNH, WM }\end{array}$ & $\begin{array}{l}\text { Type: AT (stationary bicycle), ST (hand and ankle weights), } \\
\text { stretching, relaxation } \\
\text { Duration: } 4-6 \text { weeks } \\
\text { Intensity and volume: AT } 5 \text { times a week Low to moderate } \\
\text { (HRmax } 50-75 \% \text { of RPE } 10 / 13 \text { ), stretching (Dynamic: } 1-2 \\
\text { sets of } 10-12 \text { reps; Static: } 1 \text { set/15-30 sg), ST } 3 \text { times a week } \\
\text { low to moderate, (1-2 sets of } 10-12 \text { reps at RPE 10/13) and } \\
\text { relaxation twice a week (Low RPE } 6 / 9 \text { ) } \\
\text { Cadence: } 30-70 \text { cycles/min and range of } 30-75 \mathrm{~W} \text {. } \\
\text { Volume: ST, stretching and relaxation (Dynamic: (1-2 sets of } \\
\text { 10-12 reps); Static: (1 set } 15-30 \text { sg) } \\
\text { Supervised: yes } \\
\text { Setting: Hospital }\end{array}$ & $\begin{array}{l}\text { T1-T2: } \\
-\mathrm{VO}_{2}: \uparrow \text { EXP } / \downarrow \mathrm{CT} \\
(\Delta 3.1 \% ; \Delta-28.6 \%) \\
\text { - Chest press: } \uparrow \text { EXP } / \downarrow \mathrm{CT} \\
(\Delta 5.3 \% ; \Delta-18.6 \%) \\
\text { - Leg extension: } \uparrow \text { EXP } / \downarrow \mathrm{CT} \\
(\Delta 4.3 \% ; \Delta-30.3 \%) \\
\text { - Right elbow flexor: } \uparrow \text { EXP } / \downarrow \\
\text { CT } \\
\text { ( } \Delta 6.4 \% ; \Delta-23.1 \%) \\
- \text { Right knee extensor: } \uparrow \\
\text { EXP } / \downarrow \mathrm{CT} \\
(\Delta 3.5 \% ;(\Delta-21.3 \%) \\
-2 \mathrm{MWT}: \downarrow \text { EXP } / \downarrow \mathrm{CT} \\
(\Delta-26.0 \% ; \Delta-11.3 \%) \\
-\leftrightarrow \text { Immune system }\end{array}$ \\
\hline
\end{tabular}


Table 2. Cont.

\begin{tabular}{|c|c|c|c|c|c|}
\hline Study & Study Design & $\begin{array}{l}\text { Sample Size by Group (Sex), Age } \\
\text { (Mean } \pm \text { SD; Range) }\end{array}$ & Primary Cancer & Intervention & $\begin{array}{l}\text { Main Results in Physical } \\
\text { Related Variables }\end{array}$ \\
\hline $\begin{array}{l}\text { (Wiskemann et al., } \\
\text { 2014) [45] }\end{array}$ & $\begin{array}{l}\text { Multicenter RCT } \\
\text { T1: Pre HSCT (baseline)-Pre } \\
\text { HSCT (A) } \\
\text { T2: Pre HSCT (A)-Post HSCT } \\
\text { (D) } \\
\text { T3: Post HSCT (D)-Post } \\
\text { HSCT (6-8 weeks) }\end{array}$ & $\begin{array}{l}\text { - EXP: } n=52,(21 \text { female), } \\
47.6 \text { years (18-70) } \\
\text { - CT: } n=53 \text {, (13 female), } 50 \text { years } \\
(20-71)\end{array}$ & $\begin{array}{l}\text { AA, ALL, AML, CLL, } \\
\text { CML, Lymphoma, MDS, } \\
\text { MM }\end{array}$ & $\begin{array}{l}\text { Type: AT (N/R), ST (elastic bands) } \\
\text { Duration: } 8 \text { weeks. } \\
\text { Frequency: AT: T1 ( } 3 \text { times a week), T2 (5 times a week), T3 } \\
\text { ( } 3 \text { times a week). ST: T1, T2, and T3 (twice a week). } \\
\text { Intensity and volume: Not specified } \\
\text { Supervised: yes } \\
\text { Setting: home/hospital }\end{array}$ & $\begin{array}{l}\text { T1-T3: EXfit: } \\
-\downarrow \text { Knee extensión } \\
(\Delta-31.3 \%) \\
-\downarrow \text { Hip flexion } \\
(\Delta-16.4 \%) \\
-\downarrow \text { Elbow extensión } \\
(\Delta-21.2 \%) \\
-\downarrow \text { Elbow flexion } \\
(\Delta-22.1 \%) \\
-\downarrow 6 \mathrm{MWT} \\
(\Delta-4.3 \%) \\
\text { T1-T3: Exunfit: } \\
-\downarrow \text { Knee extensión } \\
(\Delta-8.6 \%) \\
-\uparrow \text { Hip flexion } \\
(\Delta 10.3 \%) \\
-\uparrow \text { Elbow extensión }(\Delta 3.4 \%) \\
-\downarrow \text { Elbow flexion } \\
-\uparrow 6 \mathrm{MWT}(\Delta 10.4 \%) \\
-\leftrightarrow \text { Immune system }\end{array}$ \\
\hline $\begin{array}{l}\text { (Jarden et al., } \\
\text { 2007) [31] }\end{array}$ & $\begin{array}{l}\text { RCT } \\
\text { T1: Pre HSCT (A) } \\
\text { T2:Post HSCT } \\
( \pm 1 \text { day D) }\end{array}$ & $\begin{array}{l}\text { - EXP: } n=6 \text { ( } 2 \text { female), } 34 \text { years } \\
(18-58) \\
- \text { CT: } n=8 \text { (4 female) } 37 \text { years } \\
(18-53)\end{array}$ & $\begin{array}{l}\text { AA, ALL, AML, CML, } \\
\text { hemoglobinuria, LHN, MF, } \\
\text { MM, PNH }\end{array}$ & $\begin{array}{l}\text { Type: AT (stationary bicycle), ST (hand and ankle weights), } \\
\text { stretching, relaxation } \\
\text { Duration: } 6 \text { weeks } \\
\text { Intensity and volume: AT } 5 \text { times a week (Low to moderate } \\
50-75 \% \text { HR max and RPE } 10-13 / 20 \text { ), ST } 3 \text { times a week (Low } \\
\text { to moderate } 1-2 \text { sets of } 10-12 \text { reps of } 1 \text { RM at RPE } 10-13 / 20 \text { ), } \\
\text { stretching } 5 \text { times a week (Dynamic: } 1-2 \text { sets of } 10-12 \text { reps } \\
\text { Static: } 1 \text { set, hold for } 15-30 \text { s), relaxation } 2 \text { times a week } \\
\text { (Low RPE 6-9/20), Psychoeducation } 5 \text { times a week } \\
\text { Cadence: } 30-70 \text { cycles since } 50 \text { W } \\
\text { Supervised: yes } \\
\text { Setting: hospital }\end{array}$ & $\begin{array}{l}\text { T1-T2: } \\
\text { - Chest press: } \downarrow \text { CT } \\
(\Delta-14.9 \%) \\
\text { - Leg ext: } \uparrow \text { EXP } / \downarrow \text { CT } \\
\text { ( } \Delta 6.1 \% ; \Delta \text { - } 16.3 \%) \\
\text { - Right knee flex: } \uparrow \text { EXP } / \downarrow C T \\
(\Delta 0.2 \% ; \Delta \text { - } 14.5 \%) \\
\text { - } \leftrightarrow \text { Immune system }\end{array}$ \\
\hline
\end{tabular}


Table 2. Cont.

\begin{tabular}{|c|c|c|c|c|c|}
\hline Study & Study Design & $\begin{array}{l}\text { Sample Size by Group (Sex), Age } \\
\text { (Mean } \pm \text { SD; Range) }\end{array}$ & Primary Cancer & Intervention & $\begin{array}{l}\text { Main Results in Physical } \\
\text { Related Variables }\end{array}$ \\
\hline $\begin{array}{l}\text { (Mello et al., } \\
\text { 2003) [46] }\end{array}$ & $\begin{array}{l}\text { RCT } \\
\text { T1: Pre HSCT (A)-Post HSCT } \\
\text { (D) } \\
\text { T2: Post HSCT } \\
\text { (6 weeks) }\end{array}$ & $\begin{array}{l}\text { - EXP: } n=9,(4 \text { female), } 27.9 \text { years } \\
(18-39) \\
- \text { CT: } n=9,(6 \text { female), } 30.2 \text { years } \\
(18-44)\end{array}$ & $\begin{array}{l}\text { AML, CML, LHN, MDS, } \\
\text { SAA }\end{array}$ & $\begin{array}{l}\text { Type:AT (walking), stretching, mobilization } \\
\text { Duration: } 6 \text { weeks } \\
\text { Volume: AT, range of motion and stretching ( } 5 \text { sets of } 3 \text { min } \\
\text { comfortable walk to } 2 \text { sets of } 10-20 \text { min at } 70 \% \mathrm{HR} \text { max } \\
\text { speed walk in the sixth week) } \\
\text { Rest: } 3 \text { min } \\
\text { Supervised: yes } \\
\text { Setting: hospital }\end{array}$ & $\begin{array}{l}\text { T1-T2: } \\
\text { - Knee DM-NDM Flexors: } \downarrow \\
\text { EXP/ } \downarrow \text { CT } \\
\text { - Shoulder:DM-NDM: } \downarrow \text { CT } \\
\text { - Elbow DM flexors/NDM: } \downarrow \\
\text { CT } \\
\text { - Ankle DM/NDM Flexors: } \downarrow \\
\text { CT } \\
\text { - } \leftrightarrow \text { Immune system }\end{array}$ \\
\hline $\begin{array}{l}\text { (Wiskemann et al., } \\
\text { 2015) [47] }\end{array}$ & $\begin{array}{l}\text { Multicenter RCT } \\
\text { T1: Pre HSCT (A) } \\
\text { T2: Post HSCT (D) }\end{array}$ & $\begin{array}{l}\text { - EXP: } n=50,(21 \text { female }) \\
48.2 \pm 14.5^{\mathrm{b}} \text { years } \\
\text { - CT: } n=53,(13 \text { female }) \\
50 \pm 12.4^{\text {b years }}\end{array}$ & $\begin{array}{l}\text { AA, ALL, AML, CLL, } \\
\text { CML, Lymphoma, MDS, } \\
\text { MM }\end{array}$ & $\begin{array}{l}\text { Type: AT (walking, stationary bicycle), ST (elastic bands) } \\
\text { Duration: } 15-18 \text { weeks } \\
\text { Volume and Intensity: AT 3-5 times a week (RPE 12-14/20), } \\
\text { ST twice a week (RPE 12-14/20) } \\
\text { Supervised: yes } \\
\text { Setting: home/hospital }\end{array}$ & $\begin{array}{l}\text { T1-T2: } \\
\text { - No changes }\end{array}$ \\
\hline $\begin{array}{l}\text { (Peters et al., } \\
\text { 2018) [48] }\end{array}$ & $\begin{array}{l}\text { RCT } \\
\text { T1: Pre HSCT (H) } \\
\text { T2: Post HSCT (D) } \\
\text { T3: Post HSCT (6 weeks) }\end{array}$ & $\begin{array}{l}n=70,53.1 \pm 13.5 \text { years } \\
\text { - EXP: } n=37 \text { ( } 15 \text { female) } \\
\text { - CT: } n=33 \text { (13 female) }\end{array}$ & $\begin{array}{l}\text { ALL, AML, CLL, CML, HL, } \\
\text { LHN, MDS, MM }\end{array}$ & $\begin{array}{l}\text { Type: ST (elastic bands), range of motion } \\
\text { Duration: } 6 \text { weeks } \\
\text { Intensity: ST T2-T3 (RPE 13-14/20) } \\
\text { Intensity and volume: ST and range of motion: T2-T3 three } \\
\text { times a week for } 16 \text { weeks with } 18 \text { sessions (1-2 sets of } \\
\text { increase reps with bands) } \\
\text { Supervised: not T1-T2 and yes T2-T3 } \\
\text { Setting: hospital/home }\end{array}$ & - No changes \\
\hline $\begin{array}{l}\text { (Pahl et al., } \\
\text { 2018) [49] }\end{array}$ & $\begin{array}{l}\text { RCT pilot study } \\
\text { T1: Pre HSCT (Admission) } \\
\text { T2: Post HSCT (D) }\end{array}$ & $\begin{array}{l}\text { - EXP: } n=6 \text { ( } 1 \text { female), } 47 \text { years } \\
(19-62) \\
- \text { CT: } n=5 \text { ( } 2 \text { female), } 56 \text { years } \\
(32-63)\end{array}$ & $\begin{array}{l}\text { ALL, AML, APL, HL, } \\
\text { LHN, MM, MW, PMF }\end{array}$ & $\begin{array}{l}\text { Type: AT stationary bicycle (CT), and ST with vibration } \\
\text { (EXP) } \\
\text { Duration: } 27 \text { days } \\
\text { Intensity: AT and ST (RPE 14-16/20) } \\
\text { Intensity and Volume: AT (CT) (20 min with/without rest), } \\
\text { ST (EXP) 3times a week ( } 3 \text { sets, 30-60 sg) } \\
\text { Rest: ST: } 30-60 \text { sg (between exercises), 60-120 sg } \\
\text { (between sets) } \\
\text { Supervised: yes } \\
\text { Setting: Hospital }\end{array}$ & $\begin{array}{l}\text { T1-T2: } \\
-\uparrow \text { Jumping height }(\Delta 12.4 \%) \\
-\downarrow \text { TUG }(\Delta-19.4 \%) \\
\uparrow \text { STEO } / \uparrow \text { STEC sway path } \\
(\Delta 5.5 \%,(\Delta 7.7 \%))\end{array}$ \\
\hline
\end{tabular}


Table 2. Cont.

\begin{tabular}{|c|c|c|c|c|c|}
\hline Study & Study Design & $\begin{array}{l}\text { Sample Size by Group (Sex), Age } \\
\text { (Mean } \pm \text { SD; Range) }\end{array}$ & Primary Cancer & Intervention & $\begin{array}{l}\text { Main Results in Physical } \\
\text { Related Variables }\end{array}$ \\
\hline $\begin{array}{l}\text { (Pahl et al., } \\
\text { 2020) [54] }\end{array}$ & $\begin{array}{l}\text { RCT } \\
\text { T1: Pre HSCT (Admission) } \\
\text { T2: Post HSCT (D) } \\
\text { T3: PostHSCT (180 days D) }\end{array}$ & $\begin{array}{l}\text { - EXP: } n=18 \text { ( } 7 \text { female), } 55 \text { years } \\
(50-63) \\
- \text { CT: } n=26 \text { (7 female), } 56 \text { years } \\
(32-63)\end{array}$ & $\begin{array}{l}\text { ALL, AML, CLL, CML, } \\
\text { Lymphoma, MDS, MF, } \\
\text { MM, SAA, Septic } \\
\text { granulomatosis, }\end{array}$ & $\begin{array}{l}\text { Type: ST with vibration (EXP), mobilization of the spine and } \\
\text { stretching (CT) } \\
\text { Duration: N/R } \\
\text { Intensity: N/R } \\
\text { Volume: WBV/ST (EXP) 5times a week ( } 20 \text { min), } \\
\text { mobilization and stretching }(C T) 5 \text { times a week }(20 \mathrm{~min}) \\
\text { Supervised: yes } \\
\text { Setting: Hospital }\end{array}$ & $\begin{array}{l}\text { T1-T3: } \\
-\mathrm{VO}_{2}: \uparrow \operatorname{EXP} / \downarrow \mathrm{CT} \\
(\Delta 5.3 \% ;(\Delta-11.8 \%) \\
\text { - P max: } \uparrow \operatorname{EXP}(\Delta 16.3 \%) \\
\text { - Knee flexors/extensors: } \downarrow \\
\mathrm{CT} \\
(\Delta-23.1 \% ; \Delta-3.9 \%) \\
-\mathrm{JH}: \downarrow \mathrm{CT}(\Delta-3.3 \%) \\
\text { - BMI: } \downarrow \text { EXP } / \downarrow \mathrm{CT} \\
(\Delta-3.1 \% ;(\Delta-12.4 \%)\end{array}$ \\
\hline $\begin{array}{l}\text { (Almeida et al., } \\
\text { 2020) [55] }\end{array}$ & $\begin{array}{l}\text { RCT } \\
\text { T1: Pre HSCT (Admission) } \\
\text { T2: Post HSCT (D) }\end{array}$ & $\begin{array}{l}\text { - EXP: } n=15 \text { ( } 7 \text { female), } 46.6 \text { years } \\
(35.1-52) \\
\text { - CT: } n=16(6 \text { female), } \\
\text { years } 37.5 \text { ( } 39.5-53.6)\end{array}$ & $\begin{array}{l}\text { AML, Amyloidosis, HL, } \\
\text { LHN, MM, SAA }\end{array}$ & $\begin{array}{l}\text { Type: IMT (EXP) and AT (stationary bicycle), ADL, } \\
\text { stretching, coordination, and balance (EXP/CT) } \\
\text { Duration: N/R } \\
\text { Intensity: IMT ( } 40 \% \text { MIP) } \\
\text { Volume: IMT (EXP) } 5 \text { times a week (10-20 min, 12-16 } \\
\text { diaphragmatic breathing per min), AT (EXP/CT) } 5 \text { times a } \\
\text { week (10-20 min at 50-70\% HR max), ADL weekends } \\
\text { (EXP/CT), stretching (EXP) (CT) } 5 \text { times a week (10-20 min) } \\
\text { Supervised: yes } \\
\text { Setting: Hospital }\end{array}$ & $\begin{array}{l}\text { T1-T2: } \\
\text { - MIP: } \uparrow \text { EXP } \\
(\Delta 19.3 \%)\end{array}$ \\
\hline
\end{tabular}

Abbreviations: A = admission; $\mathrm{AA}=$ aplastic anemia; $\mathrm{ADL}=$ activities of daily living, $\mathrm{AML}=$ acute myeloid leukemia; $\mathrm{ALL}=$ acute lymphoid leukemia; $\mathrm{APL}=$ acute promyelocytic leukemia; $\mathrm{AT}=$ aerobic training; $\mathrm{BMI}=$ body mass index; $\mathrm{CLL}=$ chronic lymphocytic leukemia; $\mathrm{CML}=$ chronic myeloid leukemia; $\mathrm{CR}=$ chair-rising test; $\mathrm{CRF}=$ cardiorespiratory fitness; $\mathrm{CRT}=$ chair-rising test; $\mathrm{CT}=$ control group; $\mathrm{D}=$ discharge; $\mathrm{DCT}=$ daily cardiovascular training; $\mathrm{EXP}=$ experimental group; $\mathrm{EX}$ fit = initially fit patients in the exercise group; $\mathrm{EX}$ unfit = initially unfit patients in the exercise group; Ext = extension; FRM = fat-free mass; Flex = flexion; $\mathrm{H}=$ hospitalization; Hb = hemoglobin; HL = Hodgkin's lymphoma; HRmax = heart rate maximal; HSCT = hematopoietic stem cell transplantation; IMT = inspiratory muscle training; JH = jump height; LBW = lean body weight; LHN = non-Hodgkin's lymphoma; KPS = Karnofsky Performance Status; MDS = myelodysplastic síndrome; MIP = maximal inspiratory pressure; MF = myelofibrosis; $\mathrm{MM}=$ múltiple mieloma; $\mathrm{MPS}=$ myeloproliferative síndrome; MW = Morbus Waldenström; PCMJ = power output during counter-movement jump; PID = primary immune deficiency; Plts = platelets; PMF = primary myelofibrosis Pmax = maximum power output; $\mathrm{PNH}=$ paroxysmal nocturnal hemoglobinuria; $\mathrm{RCT}=$ randomized controlled trial; $\mathrm{RPE}=$ rate of perceived exertion; $\mathrm{ST}=$ strength training $\cdot \mathrm{SAA}=$ timed up and go test $(3 \mathrm{~m}) ; \mathrm{VO}_{2}=$ oxygen uptake; $\mathrm{WBV}=$ whe 30CST = 30-s chair stand test. Symbols: $\leftrightarrow=$ no improvement; $\uparrow=$ increase; $\downarrow=$ decrease; Min = minutes; $\mathrm{n}=$ sample size; $\mathrm{N} / \mathrm{R}=$ not reported; Reps = repetitions; $\mathrm{Sg}=$ seconds; $1 \mathrm{RM}=1$ repetition máximum; ${ }^{\mathrm{a}}($ median $\pm \mathrm{SD}){ }^{\mathrm{b}}($ mean $\pm \mathrm{SD})$ 
Table 3. Studies that have analyzed the effects of an exercise program after HSCT, with main results in physically related variables.

\begin{tabular}{|c|c|c|c|c|c|}
\hline Study & Study Design & $\begin{array}{l}\text { Sample Size by Group (Sex), } \\
\text { Age (Mean } \pm \text { SD; Range) }\end{array}$ & Primary Cancer & Intervention & $\begin{array}{c}\text { Main Results in Physical } \\
\text { Related Variables }\end{array}$ \\
\hline $\begin{array}{l}\text { (Shelton et al., } \\
\text { 2009) [34] }\end{array}$ & $\begin{array}{l}\text { RCT } \\
\text { T1: Pre HSCT (A) } \\
\text { T2:Post HSCT (4 weeks) }\end{array}$ & $\begin{array}{l}\text { - Supervised: } n=26,(9 \text { female }) \\
43.65 \pm 13.18^{\text {a }} \text { years } \\
\text { - Self-directed: } n=27 \\
\text { (11 female), } 93 \pm 11.66^{\text {a }} \text { years }\end{array}$ & $\begin{array}{l}\text { ALL, AML, CLL, CML, } \\
\text { HD, Lymphoma, LHN }\end{array}$ & $\begin{array}{l}\text { Type: AT (stationary bicycle, treadmill), ST (weight } \\
\text { machines) } \\
\text { Duration: } 4 \text { weeks } \\
\text { Volume and intensity: AT: } 3 \text { days a week (20-30 min } \\
60-75 \% \text { HR max and BFI: } 0-10 \text { ), ST: } 3 \text { days a week } \\
\text { EXP supervised (1-3 sets of } 10 \text { reps), EXP } \\
\text { self-directed (1-3 sets of } 10-15 \text { reps). The AT and ST } \\
\text { increased every third visit; if extreme fatigue, } \\
\text { resistance was reduced to the previous level. } \\
\text { Supervised: yes } \\
\text { Setting: hospital/home }\end{array}$ & $\begin{array}{l}\text { T1-T2: } \\
\text { Supervised }= \\
-\downarrow 50 \text { FWT }(\Delta-13.7 \%) \\
\text { Self-directed }= \\
-\uparrow 6 \mathrm{MWT}(\Delta 12.0 \%) \\
-\leftrightarrow \text { Immune system }\end{array}$ \\
\hline $\begin{array}{l}\text { (Barğ1 et al., } \\
\text { 2016) [35] }\end{array}$ & $\begin{array}{l}\text { RCT } \\
\text { T1: Pre HSCT (A) } \\
\text { T2: Post HSCT (6 weeks) }\end{array}$ & $\begin{array}{l}\text { - EXP: } n=20,(8 \text { female }) \\
34.10 \pm 12.65^{\text {a }} \text { years } \\
\text { - CT: } n=18,(6 \text { female }) \\
39.11 \pm 12.57^{\text {a }} \text { years }\end{array}$ & $\begin{array}{l}\text { AA, ALL, AML, CML, } \\
\text { Fanconi anemia, MDS, } \\
\text { MM, LHN, PNH }\end{array}$ & $\begin{array}{l}\text { Type: Respiratory muscle } \\
\text { Duration: } 6 \text { weeks } \\
\text { Volume and intensity: AT: } 7 \text { days at week (speed } \\
\text { progressively increased at } 1 \text { min intervals walking at } \\
12 \text { stages } / 30 \text { min rest between } 2 \text { tests with FIS (1-4). } \\
\text { Diaphragmatic breaths: } 7 \text { days at week (EXP) (15 } \\
\text { sg/25-30 breaths } / 5-10 \text { resting IMT at } 40 \% \text { of MIP), } \\
\text { (CT) (received sham IMT at fixed workload, 5\% of } \\
\text { baseline MIP with MMRC (0-4)) } \\
\text { Supervised: yes } \\
\text { Setting: hospital/home }\end{array}$ & $\begin{array}{l}\text { T1-T2: } \\
-\uparrow \operatorname{MISWT}(\Delta 8.0 \%) \\
-\uparrow 6 \mathrm{MWT}(\Delta 5.8 \%) \\
-\uparrow \operatorname{MIP}(\Delta 36.6 \%) \\
-\mathrm{MEP}: \uparrow \mathrm{EXP} / \uparrow \mathrm{CT} \\
(\Delta 15.3 \%) \\
-\mathrm{FEV} 1 / \mathrm{FVC}: \downarrow \mathrm{CT} \\
(\Delta-1.1) \\
-\downarrow \operatorname{MMRC}(\Delta-37.5 \%) \\
-\leftrightarrow \operatorname{Imm} \% \text { system }\end{array}$ \\
\hline $\begin{array}{l}\text { (KIM \& KIM, } \\
2006)[41]\end{array}$ & $\begin{array}{l}\text { RCT } \\
\text { T1: Pre HSCT (A) } \\
\text { T2: Post HSCT (6 weeks) }\end{array}$ & $\begin{array}{l}\text { - EXP: } n=18 \text { (10 female), } \\
32.9 \pm 7.0^{\text {a }} \text { years } \\
\text { - CT: } n=17 \text { ( } 8 \text { female }) \\
34.3 \pm 7.8^{\text {a }} \text { years }\end{array}$ & $\mathrm{AA}, \mathrm{ALL}, \mathrm{AML}$ & $\begin{array}{l}\text { Type: bed exercise intervention: Stretching, mobility, } \\
\text { relaxation breathing } \\
\text { Duration: } 6 \text { weeks } \\
\text { Volume and intensity: Stretching and mobility bed } \\
\text { exercise intervention: } \\
7 \text { days a week, } 3 \times 10 \text { min: preliminary exercise. } \\
\text { Relaxation breathing and finish exercise. } \\
\text { Supervised: yes } \\
\text { Setting: hospital }\end{array}$ & $\begin{array}{l}\text { T1-T2: } \\
\text { Lymphocytes: } \downarrow \text { CT }\end{array}$ \\
\hline
\end{tabular}


Table 3. Cont.

\begin{tabular}{|c|c|c|c|c|c|}
\hline Study & Study Design & $\begin{array}{c}\text { Sample Size by Group (Sex), } \\
\text { Age (Mean } \pm \text { SD; Range) }\end{array}$ & Primary Cancer & Intervention & $\begin{array}{c}\text { Main Results in Physical } \\
\text { Related Variables }\end{array}$ \\
\hline $\begin{array}{l}\text { (Persoon et al., } \\
\text { 2017) [50] }\end{array}$ & $\begin{array}{l}\text { RCT } \\
\text { T1: Post HSCT (A) } \\
\text { T2: Post HSCT (18 weeks) }\end{array}$ & $\begin{array}{l}\text { - EXP: } n=54 \text { (22 female), } \\
53.5 \text { years (20-67) } \\
\text { - CT: } n=55 \text { (18 female), } \\
56 \text { years (19-67) }\end{array}$ & HL, MM & $\begin{array}{l}\text { Type: AT (stationary bicycle), ST (weight machines) } \\
\text { Duration: } 18 \text { weeks } \\
\text { Intensity and volume: AT 1-8 week twice a week } \\
\text { (blocks of } 30 \text { sg at } 65 \% \text { MSEC alternated with blocks } \\
\text { of } 60 \text { s at } 30 \% \text { MSEC), from } 9-18 \text { weeks (blocks of } 30 \text { s } \\
\text { at } 65 \% \text { MSEC alternated with blocks of } 30 \text { sg at } 30 \% \\
\text { MSEC), ST } 1-12 \text { weeks twice a week ( } 2 \text { sets } / 10 \text { reps } \\
60-80 \% 1 \text { RM, from 13-18 weeks once a week (1 } \\
\text { set } / 20 \text { reps } 35-40 \% 1 \text { RM). } \\
\text { Supervised: Yes } \\
\text { Setting: local physiotherapy }\end{array}$ & $\begin{array}{l}\text { T1-T2: } \\
\text { - No changes }\end{array}$ \\
\hline $\begin{array}{l}\text { (Bird et al., } \\
\text { 2010) [51] }\end{array}$ & $\begin{array}{l}\text { RCT } \\
\text { T1: Post HSCT (A) } \\
\text { T2: Post HSCT (6 months) }\end{array}$ & $\begin{array}{l}\text { - EXP: } n=29 \text { (13 female), } \\
57 \text { years (44-53.5) } \\
\text { - CT: } n=29 \text { (7 female), } 52 \text { years } \\
(42.5-63)\end{array}$ & $\begin{array}{l}\text { Leukaemia, Lymphoma, } \\
\text { Myeloma }\end{array}$ & $\begin{array}{l}\text { Type: AT, relaxation } \\
\text { Duration: } 10 \text { weeks } \\
\text { Intensity and volume: AT CEXP) 1-10 weeks (a series } \\
\text { of circuit training exercise), relaxation (guided } \\
\text { imagery). AT (CT) 1-10 weeks } 3 \text { times/week } \\
\text { (home-based exercise program) } \\
\text { Supervised: Yes } \\
\text { Setting: Hospital/Home }\end{array}$ & $\begin{array}{l}\text { T1-T2: } \\
\text { - No changes }\end{array}$ \\
\hline $\begin{array}{l}\text { (Oechsle et al., } \\
\text { 2014) [52] }\end{array}$ & $\begin{array}{l}\text { RCT } \\
\text { T1: Post HSCT (A) } \\
\text { T2: Post HSCT } \\
\text { (After intervention) }\end{array}$ & $\begin{array}{l}\text { - EXP: } n=17 \text { (7 female), years } \\
51.7 \pm 13.3^{\mathrm{c}} \\
\text { - CT: } n=17(7 \text { female), years } \\
52.9 \pm 15.4^{\mathrm{c}}\end{array}$ & $\begin{array}{l}\text { AML, LHN, MM, Germ } \\
\text { cell tumor }\end{array}$ & $\begin{array}{l}\text { Type: AT (stationary bicycle), ST (elastic bands, } \\
\text { bodyweight) } \\
\text { Duration: } 21 \text { days } \\
\text { Intensity and volume: AT } 5 \text { times a week }(10-20 \mathrm{~min}) \text {, } \\
\text { ST } 5 \text { times a week ( } 20 \mathrm{~min}, 2 \text { sets of } 16-25 \text { reps at } \\
40-60 \% \text { of } 1 \text { RM) } \\
\text { Rest: AT (regular pauses until recuperated to } 66.6 \%) \\
\text { Supervised: Yes } \\
\text { Setting: Hospital }\end{array}$ & $\begin{array}{l}\text { T1-T2: } \\
\text { - CT: } \downarrow \mathrm{VO}_{2}(\Delta-26.0 \%) \\
\text { - EXP: } \uparrow \mathrm{VO}_{2}, \mathrm{VE} \text { and } \\
\text { strength upper limbs } \\
(\Delta 11.3 \% ;(\Delta 21.8 \% ; \\
(\Delta 35.7 \%)\end{array}$ \\
\hline
\end{tabular}


Table 3. Cont.

\begin{tabular}{|c|c|c|c|c|c|}
\hline Study & Study Design & $\begin{array}{l}\text { Sample Size by Group (Sex), } \\
\text { Age (Mean } \pm \text { SD; Range) }\end{array}$ & Primary Cancer & Intervention & $\begin{array}{c}\text { Main Results in Physical } \\
\text { Related Variables }\end{array}$ \\
\hline $\begin{array}{l}\text { (Hacker et al., } \\
\text { 2011) [37] }\end{array}$ & $\begin{array}{l}\text { RCT } \\
\text { T1: Pre HSCT-Post-HSCT } \\
\text { (After } 8^{\circ} \text { day) } \\
\text { T2: H-Post-HSCT } \\
\text { (1-6 week) }\end{array}$ & $\begin{array}{l}\text { - EXP: } n=9 \\
\text { - CT: } n=10 \\
n=19,(5 \text { female }) 46.26 \text { years } \\
(16.23)^{\mathrm{c}}\end{array}$ & AML, Lymphoma & $\begin{array}{l}\text { Type: ST (elastic bands, bodyweight) } \\
\text { Duration: } 6 \text { weeks } \\
\text { Volume and intensity: ST } 1-6 \text { weeks } 3 \text { times a week } \\
\text { (1-2 sets of } 8-10 \text { reps of RPE }(13 / 20) \\
\text { Supervised: yes } \\
\text { Setting: hospital/home }\end{array}$ & $\begin{array}{l}\text { T2: } \\
-\uparrow 30 \mathrm{CST}: \text { EXP/CT } \\
(\Delta-12.7 \% ; \Delta-12.0 \%) \\
-\downarrow \text { TNSU: EXP/CT } \\
(\Delta-18.9 \% ; \Delta-20.3 \%) \\
-\leftrightarrow \text { Immune system }\end{array}$ \\
\hline $\begin{array}{l}\text { (Knols et al., } \\
\text { 2011) [43] }\end{array}$ & $\begin{array}{l}\text { RCT } \\
\text { T1: Pre HSCT (A)-Post } \\
\text { HSCT (D) } \\
\text { T2: Post HSCT (D)-Post } \\
\text { HSCT (3 months) }\end{array}$ & $\begin{array}{l}\text { - EXP: } n=64 \text { (26 female), } \\
46.7 \pm 13.7 \text { years }(18-75)^{\text {a }} \\
\text { - CT: } n=67(28 \text { female }), \\
46.6 \pm 12 \text { years }(20-67)^{a}\end{array}$ & $\begin{array}{l}\text { ALL, AML, } \\
\text { Amyloidosis, CLL, HL, } \\
\text { LHN, Lymphoma, MM, } \\
\text { osteomyelofibrosis, } \\
\text { testicular cancer }\end{array}$ & $\begin{array}{l}\text { Type: AT (stationary bicycle), ST (dumbbell) } \\
\text { Frequency: } 2 \text { days/week } \\
\text { Duration: } 12 \text { weeks } \\
\text { Intensity and volume: AT twice a time (50-70\% to 80\% } \\
\text { HRmax) } \\
\text { Supervised: yes } \\
\text { Setting: fitness center/physiotherapy practise }\end{array}$ & $\begin{array}{l}\text { T1-T2: } \\
\text { - Knee extensión: } \uparrow \text { EXP } / \uparrow \\
\text { CT }(\Delta 26.2 \%) \\
\text { - Walk speed: } \downarrow \text { EXP } / \downarrow \mathrm{CT} \\
(\Delta-11.9 \% ; \Delta-2.4 \%) \\
\text { - } 6 \mathrm{MWT}: \uparrow \mathrm{EXP} / \uparrow \mathrm{CT} \\
(\Delta 17.7 \% ; \Delta 9.8 \%) \\
\text { - } \leftrightarrow \text { Immune system }\end{array}$ \\
\hline $\begin{array}{l}\text { (Schumacher } \\
\text { et al., 2018) [44] }\end{array}$ & $\begin{array}{l}\text { RCT } \\
\text { T1: Pre HSCT (A)-Post } \\
\text { HSCT (14 days) } \\
\text { T2: Pre HSCT (A)-Post } \\
\text { HSCT (30 days) }\end{array}$ & $\begin{array}{l}\text { - EXP: } n=19(3 \text { female), } \\
56 \text { years }(21-65) \text { b } \\
\text { - CT: } n=23(14 \text { female), } \\
56.5 \text { years }(23-69)^{b}\end{array}$ & $\begin{array}{l}\text { AML, CLL, CML, LHN, } \\
\text { MDS, MM, Teratoma }\end{array}$ & $\begin{array}{l}\text { Type: AT (walking, step), ST (elastic bands, } \\
\text { bodyweight), Stretching, Wii sports, Wii fit program, Wii } \\
\text { balance } \\
\text { Duration: } 100 \text { days } \\
\text { Intensity: N/R } \\
\text { Frequency: } 5 \text { days/week } \\
\text { Supervised: yes } \\
\text { Setting: hospital }\end{array}$ & $\begin{array}{l}\text { T1-T2: } \\
\text { - 2MWT: } \downarrow \text { EXP } / \downarrow \text { CT } \\
(\Delta-1.7 \% ; \Delta-4.9 \%) \\
\text { - Treadmill: } \downarrow \text { EXP } / \downarrow \text { CT } \\
(\Delta-13.2 \% ; \Delta-3.8 \%) \\
\text { - L HGS: } \\
\downarrow \text { EXP } / \downarrow \text { CT } \\
(\Delta-9.3 \% ; \Delta-8.1 \%) \\
-\leftrightarrow \text { Immune system }\end{array}$ \\
\hline
\end{tabular}


Table 3. Cont.

\begin{tabular}{|c|c|c|c|c|c|}
\hline Study & Study Design & $\begin{array}{l}\text { Sample Size by Group (Sex), } \\
\text { Age (Mean } \pm \text { SD; Range) }\end{array}$ & Primary Cancer & Intervention & $\begin{array}{c}\text { Main Results in Physical } \\
\text { Related Variables }\end{array}$ \\
\hline $\begin{array}{l}\text { (Van Dongen } \\
\text { et al., 2019) [53] }\end{array}$ & $\begin{array}{l}\text { Multicenter RCT } \\
\text { T1: Post HSCT (Baseline) } \\
\text { T2: Post HSCT } \\
\text { (After exersice or similar } \\
\text { time point in the CT) } \\
\text { T3: Post HSCT } \\
\text { (12 months later) }\end{array}$ & $\begin{array}{l}\text { - EXP: } n=54 \text { ( } 22 \text { female }) \\
52 \pm 11^{\mathrm{c}} \text { years } \\
\text { - CT: } n=55(2 \text { female }) \\
53 \pm 12^{\mathrm{c}} \text { years }\end{array}$ & $\mathrm{HL}, \mathrm{MM}$ & $\begin{array}{l}\text { Type: AT (stationary bicycle), ST (weight machines) } \\
\text { Duration: } 18 \text { weeks } \\
\text { Intensity and volume: AT } 1-8 \text { weeks twice a week ( } 2 \\
\times 8 \text { min, alternating } 30 \mathrm{sg} \text { at } 65 \% \text { and } 60 \mathrm{sg} \text { at } 30 \% \\
\text { MSEC), } 9-12 \text { weeks twice a week }(2 \times 8 \text { min, } \\
\text { alternating } 30 \text { sg at } 65 \% \text { and } 30 \mathrm{sg} \text { at } 30 \% \text { MSEC), } \\
13-18 \text { weeks once a week }(2 \times 8 \text { min, alternating } 30 \mathrm{~s} \\
\text { at } 65 \% \text { and } 30 \text { sg at } 30 \% \text { MSEC), ST: } 1-12 \text { weeks twice } \\
\text { a week ( } 2 \text { sets of } 10 \text { reps at } 65-80 \% \text { of } 1-\mathrm{RM}), 13-18 \\
\text { weeks once a week ( } 2 \text { sets of } 20 \text { reps at } 35-40 \% \text { of } \\
\text { 1-RM) } \\
\text { Supervised: Yes } \\
\text { Setting: Hospital }\end{array}$ & $\begin{array}{l}\text { T1-T3: } \\
\text { - No changes }\end{array}$ \\
\hline
\end{tabular}

Abbreviations: $\mathrm{A}=$ admission; $\mathrm{AA}=$ aplastic anemia; $\mathrm{AML}=$ acute myeloid leukemia; $\mathrm{ALL}=$ acute lymphoid leukemia; $\mathrm{AT}=$ aerobic training; $\mathrm{CLL}=$ chronic lymphocytic leukemia; $\mathrm{CML}=$ chronic myeloid leukemia; $\mathrm{CRF}=$ cardiorespiratory fitness; $\mathrm{CT}=$ control group; $\mathrm{D}=$ discharge; $\mathrm{EXP}=$ experimental group; $\mathrm{FEV} 1 / \mathrm{FVC}=$ forced expiratory volume in the first second/forced vital capacity; FIS = fatigue impact scale; HD = Hodgkin's disease; HGS = hand grip strength; HL = Hodgkin's lymphoma; HSCT = hematopoietic stem cell transplantation; LHN = non-Hodgkin's lymphoma; MDS = myelodysplastic síndrome; MEP = maximal expiratory pressure; MIP = maximal inspiratory pressure; MISWT = modified incremental shuttle walking test; MM = múltiple mieloma; MMRC = Modified Medical Research Council $\cdot$ MSEC = the maximal short exercise capacity; PASE = Physical Activity Scale for the Elderly, $\mathrm{PNH}=$ paroxysmal noctural sample size; $\mathrm{N} / \mathrm{R}=$ not reported; Reps = repetitions; $\mathrm{Sg}=$ seconds; $1 \mathrm{RM}=1$ repetition máximum; ${ }^{\mathrm{a}}$ (median $\pm \mathrm{SD} ;$ range) $^{\mathrm{b}}($ median $\pm \mathrm{SD}){ }^{\mathrm{c}}(\mathrm{mean} \pm \mathrm{SD})$ 


\subsection{Quality Assessment and Publication Bias}

The quality of the 25 included studies was high (median PEDro score $=6$, range 6-10; Table 1). There were 4 articles with a score $=6,17$ articles with a score $=7-8$, and 4 articles with a score $=9-10$.

\subsection{Characteristics of Participants}

The analyzed studies included a total of 1434 oncologic patients (age range 18 to 75 years). They were distributed into an EXP with 711 patients ( 274 women) or a CT with 723 patients (250 women), although there were studies [37,39] that did not reflect the exact number of women in the experimental or control group so, there, more women could be involved. The most common cancers in the participants were leukemia, lymphoma, and myeloma.

\subsection{Characteristics of Exercise Interventions}

The characteristics of the exercise interventions (Tables 2 and 3) were very diverse.

In total, 15 studies [23,30,31,36,38-40,42,45-49,54,55] analysed the effects of an exercise program intervention in cancer patients before and after HSCT treatment, and 10 studies analysed the effects after HSCT [34,35,37,41,43,44,50-53].

Supervision: Exercise programs were supervised by researchers in 21 studies $[23,31,34,35,37,38,40-47,49-55]$ and semi-supervised in 2 studies [36,48] and 2 studies was not supervised $[30,36]$.

Frequency: A total of 13 studies (52\%) performed exercise intervention 5 times per week $[23,31,36,38,40,42,44-47,52,54,55] ; 5$ studies $(20 \%)$ performed intervention 3 times per week $[34,37,48,49,51] ; 3$ studies $(12 \%)$ increased the exercise frequency to 7 times per week $[30,35,41]$; and 3 studies $(12 \%)$ exercised twice per week [43,50,53]. Only 1 study $(4 \%)$ did not report exercise frequency [39].

Intensity training: Six studies (24\%) did not adequately report intensity exercise [30,39,41,44,45,54], two studies $(8 \%)$ offered no information regarding strength training $[34,43]$ and one study $(4 \%)$ did not report information regarding aerobic training [52]. In 10 studies $(40 \%)$, the intensity exercise was evaluated using a perceived exertion scale (RPE) $[31,36,37,39,40,42,47-49,51]$, 7 studies (32\%) reported aerobic intensity exercise using a percentage of heart rate maximal (HRmax) $[23,31,34,40,42,43,46], 3$ studies (12\%) reported intensity using a percentage of 1 repetition maximum (1RM) $[50,52,53], 3$ studies $(12 \%)$ reported aerobic intensity exercise using a percentage of power $[38,50,53]$, and 2 studies $(8 \%)$ reported the intensity exercise using a percentage of maximal inspiratory pressure (MIP) [35,55].

Duration: A total of 14 studies (56\%) lasted up to 8 weeks [23,31,34,35,37,38,40-42,45,46,48,49,52], 7 studies (28\%) lasted between 9 and 18 weeks [30,36,43,47,50,51,53], and 1 study $(4 \%)$ lasted more than 18 weeks [39]. This information was not fully reported in three studies $(12 \%)[44,54,55]$. The most common duration was six weeks, with eight studies (32\%) being performed for this duration. The duration per session ranged from 15 to $60 \mathrm{~min}[30,31,40,42,53-55]$, and the majority of the studies included interventions that lasted either between 20 to $40 \mathrm{~min}$ (seven studies (28\%) [23,35,38,41,44,45,47]) or $60 \mathrm{~min}$ (four studies $(16 \%)$ [31,40,42,53]). Overall, 11 studies did not adequately report the duration of the exercise sessions $[34,36,37,39,43,49-52,54,55]$.

Type: The majority of the studies included multicomponent exercise interventions. Nine studies (36\%) focused on strength and aerobic training [34,36,39,43,45,47,50,52,53], three studies $(12 \%)$ included strength, aerobic training, stretching and relaxation [31,40,42], two studies $(8 \%)$ included aerobic and activities of daily living (ADL) training [23,38], one study (4\%) included stretching, aerobic and mobilization training [46], one study (4\%) included inspiratory muscle training (IMT) [35], one study (4\%) included stretching, mobilization and relaxation [41], one study (4\%) included aerobic training [30], one study (4\%) included strength and mobility training [48], one study (4\%) included strength training [37], two studies (8\%) included strength training plus whole body vibration (WBV) [49,54], one 
study (4\%) included aerobic training and relaxation [51], one study (4\%) included aerobic training, strength and stretching [44], and one study included IMT, aerobic training, ADL, stretching, coordination and balance [55]. When reported, the most-used activities for aerobic training were walking and cycling. For strength training, whole-body exercises were used with or without equipment, although the most common was the use of elastic bands. In the case of respiratory muscle exercises, pressure threshold-loading devices were used. For strength training plus whole-body vibration, a vibration platform was used. For a more detailed description, please see Tables 2 and 3.

Delivery setting: Fourteen interventions (56\%) were delivered in health care settings (e.g., hospitals) [23,31,38,40-42,44,46,49,50,52-55], nine interventions (36\%) were delivered both in the participant's homes and in health care settings $[30,34-37,45,47,48,51]$, one intervention (4\%) was delivered in the participant's home [39], and one intervention (4\%) was delivered both in the physiotherapy practice and in the fitness centre [43].

\subsection{Exercise Safety-Related Adverse Events}

A total of 7 of the 25 studies (28\%) reported adverse effects. One study (4\%) reported a calf muscle strain during a non-exercise related training session [50], and two studies (8\%) reported exercise-related adverse effects [39,55], while four studies (16\%) reported adverse effects, but it was unclear whether they were due to the exercise intervention $[23,30,36,40]$.

The exercise programs seem to be safe in most of the interventions included in the present review (i.e., 23 studies), and only 2 studies from the 25 were clearly associated with adverse events $[39,55]$. Furthermore, only $0.42 \%$ of the patients ( 3 from the 711 participants in the exercise interventions) suffered these adverse events: central venous catheter rupture in an exercise test ( 1 patient; $0.14 \%$ ) [39], vomiting (1 patient; $0.14 \%$ ), and desaturation (1 patient; $0.14 \%$ ) [55].

\subsection{Compliance Rate}

A total of 12 out of 25 papers (48\%) reported the rate of adherence of their participants to exercise, with the compliance rate of the participants being: $<40 \%[30,34]$, $40-75 \%$ [37,39,54], 80-90\% [40,48,50], >90\% [31,36,55], and not specified [49] (see Tables 2 and 3 for a more detailed description of the studies).

\subsection{Endpoints and Exercise Intervention Results}

\subsubsection{Cardiorespiratory Fitness (CRF)}

CRF was analysed in 17 of the 25 studies (68\%). The following lung function variables were measured directly with a gas analyser, spirometer, and flow screen: $\mathrm{VO}_{2 \mathrm{max}}[31,42,50,52-54]$, ventilation carbon dioxide $\left(\mathrm{VCO}_{2}\right)$ [52], expiratory minute ventilation volume (VE) [52], inspiratory vital capacity (IVC) [23], vital capacity (VC) [38], forced vital capacity (FVC) [23,35,38], MIP [35,55], maximal expiratory pressure (MEP) [35], forced expiratory volume in the first second/forced vital capacity (FEV1/FVC) [35], modified medical research council (MMRC) [35], forced expiratory volume in the first second (FEV1) [35], peak expiratory flow (PEF) [35], and forced expiratory flow from 25\% to 75\% (FEF 25-75\%) [35]. Other variables measures in CRF were: 2-min stair climb test (2MWT) [31,42,44], 6-min walk test (6MWT) [34-36,43,45], shuttle walk test (SWT) [51], and walk on treadmill (min/watts) $[39,44]$. In addition, submaximal aerobic endurance [38] and relative endurance $[23,38]$, with a submaximal endurance test based on the World Health Organization recommendations (WHO), were measured.

In 11 out of 17 articles (65\%), the results indicate a significant improvement in the following variables in CRF: $\mathrm{VO}_{2 \max }[42,52,54]$, MIP [35,55] and MEP [35], MMRC [35], $6 \mathrm{MWT}[34-36,43,45]$, and relative endurance $[23,38]$.

\subsubsection{Muscle Strength and Power}

Muscle strength was analyzed in 18 of the 25 studies (72\%). Skeletal muscle strength was determined in 16 of the 25 studies (64\%): Maximal isometric voluntary strength tests 
(MIVS) were obtained from muscle groups of the upper limbs [52], hand-grip strength (HGS) $[35,37,43-45,50,53]$, abdominal muscles [50,52,53], exercises of the autochthon back and arm muscles [52], only lower extremities [54], upper and lower limbs [35,37,42-45,50,53]. Muscle power was analyzed in 2 of the 25 studies $(8 \%)$, with the following variables: jumping height $[49,54]$ and maximum power output (Pmax) [54].

In 7 of the 16 papers of skeletal muscle strength $(44 \%)$, the results indicate an improvement in the following variables: upper limb muscle strength $[39,46,52]$, right elbow flexor [42], elbow extension [45], chest press [42], hip flexion [45], right knee flexion [31], knee extension [42,43], leg extension [31,42], lower extremities [39,46] and Strength Training to Enhance Early Recovery (STEER) [48]. Moreover, in both studies of muscle power (100\%), the results indicate that an improvement was observed in terms of an enhancement of the jumping height [49] and Pmax in a counter-movement jump test [54].

\subsubsection{Functional Mobility State}

Functional mobility and functional state was analyzed in 8 of the 25 studies (32\%) using the following variables: modified incremental shuttle walk test (MISWT) [35], 50-foot walk test (50 FWT) [34,43], 30 s chair stand test (30 CST) [34,37,50,54], timed-up-and-go test (TUG) [49], time needed to stand up (TNSU) [37], semi-tandem stance with eyes open $\left(\mathrm{ST}_{\mathrm{EO}}\right)$ and with eyes closed $\left(\mathrm{ST}_{\mathrm{EC}}\right)$ [49], step activity monitor (SAM) [43], physical activity scale for the elderly (PASE) [50], forward reach test [34], and Karnofsky performance status (KPS) [30].

In 6 of the 8 studies (75\%), the results indicate an improvement in the following functional state variables: MISWT [35], 50 FWT [43], 30 CST [37,54], TUG [37,49], ST $\mathrm{EO}$ and $\mathrm{ST}_{\mathrm{EC}}$ sway path [49], and KPS [30].

\subsubsection{Body Composition}

Body composition was analysed in 18 of the 25 studies (72\%) using body mass index (BMI) $[23,31,35,36,38,40,42,43,45-47,49,50,52,54,55]$, lean body weight (LBW) $[38,39,43,49]$, weight [30,35,38,43,52], body height [35,38,52], fat mass [43], body fat [54], fat free mass (FFM) [43,54], body cell mass [54], and phase angle [54]. In 1 of the 18 studies (6\%), the exercise program produced a significant improvement in LBW [39].

Some of the techniques mentioned to measure body composition were the following: LBW with air-displacement plethysmography $[36,39,51]$, fat mass via dual-energy X-ray absorptiometry (DXA) [43], and body cell mass and phase angle with bioelectrical impedance analysis (BIA) $[43,54]$. All the techniques agreed that they were performed before and after transplantation.

\subsection{Immune System}

The immune system was analyzed in 3 of the 25 studies (12\%) using the following variables: leukocytes [23,42], lymphocyte count [41], platelets [23,42], and hemoglobin [23]. None of the three articles showed that the exercise program produced a significant improvement or deterioration of blood parameters. Only one study maintained normal values of hematological lymphocytes (1000-4500 cells/ $\mu \mathrm{L})$ [41]. Further, the characteristics (i.e., type of cancer and treatment) of the studies analyzed are as follows: two of the three studies $(67 \%)$ used the Allo-HSCT treatment with conditioning therapy (i.e., a combination of chemotherapy, radiotherapy and/or immunotherapy) with total body irradiation (TBI) in patients with AML, ALL, and CML [23,42]. Lastly, one of the three studies (33\%) used the HSCT-BMT treatment combined with conditioning therapy in patients with AML, and ALL [41]

\section{Discussion}

This study reviews the scientific literature on the effects of exercise programs on physical factors and safety in adult patients with cancer and HSCT-BMT. The most significant result of this systematic review is that training program interventions seem to be safe in 
adult patients with cancer and HSCT and produce significant improvements in CRF, muscle strength and power, functional mobility, and functional state. To our knowledge, there are two systematic reviews and meta-analyses, published in 2013 [56,57], which studied the effects of exercise on health factors, and both included nine studies that are also analyzed in our review. Our systematic review presents similarities with these two reviews [56,57]: (a) only RCTs published in English were included; (b) exercise interventions varied widely; (c) exercise interventions were safe and well tolerated; (d) exercise programs improved physical factors. On the other hand, our review presents some differences from these previous reviews [56,57]: (a) we did not perform a meta-analysis; (b) our review included a larger number of RCTs (25 vs. 11 and 8 studies, respectively).

\subsection{Cardiorespiratory Fitness}

Of the 25 papers, in 11 of 17 articles (65\%), improvements were observed in the measured variables of CRF. Our findings on CRF show that exercise interventions increased the performance, on average, by $6.6 \%$ (3.1-11.3\%) in the $\mathrm{VO}_{2 \max }, 9.2 \%(0.2-14.5 \%)$ in the $6 \mathrm{MWT}$, and $13.9 \%(11.0-16.7 \%)$ in relative endurance. In the case of the $6 \mathrm{MWT}$, there was a study without improvement, but it showed maintenance of the baseline values $(-0.19 \%)$ compared to a decrease in the control group (-9.9\%) [36]. The 6MWT is a predictor of morbidity and mortality as well as a measure of the patient's functional status [58], and $\mathrm{VO}_{2 \mathrm{max}}$ is the most important variable related to CRF [28]. Therefore, exercise seems to have a healthy effect on the heart, prior to and after transplantation, maintaining or even increasing the physical capacity of the individual. The exercise intervention also prevents a loss of physical performance, according to Dimeo et al. [21].

On the other hand, 6 of the 17 articles (35\%) did not observe significant improvements after the exercise interventions and, although $\mathrm{VO}_{2 \max }$ was maintained in the intervention group, it was not statistically significant $[31,50,53]$. The lack of improvements may be due to the following issues: (a) the intervention group received self-training on weekends (mean 4.5 days) during hospitalization [31]; (b) the training frequency of the physical activity program was low (i.e., twice a week over 18 weeks) [50,53]; (c) there was a lack of control and rigorous planning of the variables of the exercise program (e.g., intensity, frequency, volume) and poor compliance [50]; (d) a large number of $\mathrm{VO}_{2 \text { peak }}$ values were lost (36\%), and only $23 \%$ of the patients examined participated in the study [50]; (e) $\mathrm{VO}_{2 \max }$ was measured in patients after HSCT [53], and it is possible that this measurement does not reflect an improvement in the patient's health status, as a post-transplant exercise program does not necessarily accelerate the patient's recovery process [31,53]. However, there are studies that show that $\mathrm{VO}_{2 \max }$ not only decreases due to treatment but can also be reduced due to bed rest during the first 10 days of inactivity [59].

\subsection{Muscle Strength and Power}

Of the 25 papers, in 10 of 16 studies (62.5\%), a mean increment of $11.2 \%(3.5-26.2 \%)$ is observed in the measured variables of skeletal muscle strength. Moreover, some studies $[31,39,46]$ showed the maintenance of the initial strength values in the groups of exercise intervention, with a mean improvement of $1.4 \%(0.1-4.1 \%)$. However, the control groups in these studies showed a significant loss in their initial muscle strength capacity after the HSCT, showing a mean decrease of 5.4\% (-0.4-9.1\%). Then, the exercise program plays an important protective role over the muscle capacities during the most aggressive treatment phases. On the other hand, in two out of two studies (100\%), improvements are observed in the measured variables of power, with an average increase of $18.6 \%$ (12.4-24.8\%). Strength training improves muscle mass and reduces its catabolic wear from cancer-related treatments [60] and counteracts the weakened strength observed after HSCT [61]. For these reasons, it is clinically relevant to reinforce physical functioning before and after HSCT to prevent secondary health problems [62,63]. Medical treatment alone has been extensively researched and provides fewer benefits than its implementation with an exercise program [64]. 
In contrast, in 6 of the 16 articles (37.5\%), no significant improvements in skeletal strength were observed after the exercise intervention, which may be due to the following issues in different studies: (a) a small sample size [35,37,44,54]; (b) there was a gender imbalance between semi-supervised groups $[35,37,44,50,53]$; (c) other studies did not detail some of the units of measurements on the lower limb [37,53]; (d) in the study of Persoon et al. [50], despite having a good methodological design, no improvements were reflected due to non-progressive training planning (i.e., increase of volume and intensity) and low compliance; (e) in Van Dongen's intervention, in the last 12-18 weeks, the training frequency was reduced to once a week [53]; (f) in another study with a 6-week follow-up, in which the control group was given exercise recommendations and guidelines, 4 weeks after discharge, two patients in this group exercised, and this may be a confounding factor [37]; and $(\mathrm{g})$ the IMT specific for respiratory muscles was not oriented towards gaining limb strength [35]. In another paper, no comparison was made between the WBV training and conventional resistance training during transplantation; therefore, the reported results could not be explicitly attributed to WBV training being more beneficial than conventional training [54].

\subsection{Functional Mobility and Functional State}

Of the 25 papers, in six of eight articles (75\%), a mean improvement of $13.6 \%(5.5-19.4 \%)$ was observed in the measured variables of functional mobility and functional state. In the specific case of the KPS [30], the intervention group worsened less than the control group, with a decrease of 10 points compared to the 20 points lost by the control group (KPS scale ranges from 0 to 100 points). However, in two of the eight articles [50] (25\%), no significant improvements are observed after the exercise interventions, which may be due to the following issues: (a) the time of the intervention was just after BMT [50], and there are studies that argue that exercise does not speed recovery immediately after HSCT [43]; (b) the control group was not restricted in physical activity or access to physiotherapy services, and this may have altered the results [50]; (c) the level of adherence (23\%) was very low [50]; (d) a low recruitment rate [34]; (e) the variation in the volume of physical activity between both groups [34]; (f) the lack of daily quantification of physical activity levels [34]; and (g) a program duration of only 4 weeks [34].

\subsection{Body Composition}

Of the 25 papers, in 1 of 18 articles (6\%) [39], a 0.1\% increment in LBW was observed. It should be noted that in this case, the small increase means that the exercise intervention recovered the baseline values while the control group presented LBW losses of $3.6 \%$. However, in 17 of the 18 articles (94\%), no significant improvements were observed after the exercise intervention, which may be due to the following issues: (a) the time of exercise intervention in relation to treatment, as it was applied to patients undergoing intensive chemotherapy during hospitalization, with a really high catabolic environment $[49,50]$; (b) a small sample size $[40,49,54]$; (c) difficulty in the monitoring of patients due to timetables and transport restrictions [40]; (d) the intervention was semi-supervised [40]; (e) within the sample size, the distribution of patients between both groups was not homogeneous, with a minority number of women in each group: EXP $(\mathrm{N}=64 ; 26$ female), TC $(\mathrm{N}=67$; 28 female) [43]; (f) low intensity, frequency and duration of the program in relation to the guidelines of the American College of Sports Medicine (ACSM) [43]; and (g) a drop-out rate greater than $20 \%$ [43].

\section{Limitations}

This systematic review demonstrates that the research published so far on this topic has limitations with respect to: (a) the small sample size of the groups (17 of 25 studies including < 100 participants); however, it must take into account that sometimes it may be hard to collect data from numerous patients with the same cancer type and treatment, and to meet all the inclusion and exclusion criteria; (b) the shortage of control groups [31,37,39,46,49]; 
(c) the heterogeneous characteristics of the sample (e.g., wide age range, different types of cancer, and time elapsed since diagnosis and from the end of treatment); (d) the heterogeneity of exercise interventions (e.g., different types, frequency, intensity, type of session, volume of session, type of supervision); (e) the lack or short duration of the followup [34,49,52]; and (f) the control of exercise compliance rate accomplished in only 12 out of 25 items [30,31,34,36,37,39,42,48-50,54,55]; There is low scientific evidence about the effects of exercise on the immune system of these patients, although it appears that exercise interventions have maintained normal values. However, they cannot be generalized, and it will depend on the type of cancer and the treatment administered [23,36,41,42]. Future lines of research should focus on RCTs to advance towards new exercise programs with different load planning, larger sample size, greater homogeneity of the sample, detailed control of the effect of exercise on the immune system, and long-term follow-up of the exercise program.

\section{Conclusions}

In conclusion, it seems that exercise program interventions are safe and produce benefits on CRF, muscle strength and power, and functional mobility and states in adult patients with cancer and HSCT. Thus, exercise training programs may have a cardiological and muscular protective effect as well as a healthy effect on the prevention and control of transplant complications, improving health outcomes. However, more scientific evidence, such as RCTs or meta-analyses, is still necessary to confirm these findings and improve the highest quality evidence.

Our results show that exercise program interventions are clinically relevant, and it is necessary to implement them in a generalized way in these patients. Most of the programs analyzed were supervised, multicomponent (i.e., strength, aerobic, stretching, mobilization, and activities of daily living training), 3 to 7 days a week, with a duration from 6 to 18 weeks, with a session duration of 20 to $60 \mathrm{~min}$, and developed at health care settings or at the patient's home (depending on the patient status). Further, to improve the health and quality of life benefits produced by the program, the exercise load and intensity must be individualized and controlled during the training process.

More research is needed regarding exercise tolerance in severely immunosuppressed patients, as well as research on improving body composition through concurrent exercise and nutrition interventions.

Author Contributions: A.F.S.J. and J.L.C. conceived and designed the review; E.M.-R. and T.P.-B. performed the search for articles; E.M.-R., T.P.-B., A.F.S.J. and J.L.C. extracted the results of the studies included in the review; E.M.-R. and T.P.-B. made the figures and tables; E.M.-R., T.P.-B., A.F.S.J. and J.L.C. wrote the first draft of the manuscript; E.M.-R., T.P.-B., A.F.S.J. and J.L.C. edited and revised the manuscript; A.F.S.J. and J.L.C. approved the final version of the manuscript. All authors have read and agreed to the published version of the manuscript.

Funding: This research received no external funding.

Institutional Review Board Statement: Not applicable.

Informed Consent Statement: Not applicable.

Data Availability Statement: Not applicable.

Conflicts of Interest: The authors declare no conflict of interest.

\section{References}

1. Battaglini, C.L.; Hackney, A.C.; Garcia, R.; Groff, D.; Evans, E.; Shea, T. The Effects of an Exercise Program in Leukemia Patients. Integr. Cancer Ther. 2009, 8, 130-138. Available online: http://journals.sagepub.com/doi/10.1177/1534735409334266 (accessed on 24 February 2021). [CrossRef] [PubMed]

2. Moya, R.; Espigado, I.; Parody, R.; Carmona, M.; Márquez, F.; De Blas, J.M. Evaluation of Readmissions in Hematopoietic Stem Cell Transplant Recipients. Transplant. Proc. 2006, 38, 2591-2592. [CrossRef] [PubMed] 
3. Courneya, K.S.; Keats, M.R.; Turner, A.R. Physical exercise and quality of life in cancer patients following high dose chemotherapy and autologous bone marrow transplantation. Psychooncology 2000, 9, 127-136. Available online: http://www.ncbi.nlm.nih.gov/ pubmed/10767750 (accessed on 22 August 2019). [CrossRef]

4. Weisdorf, D.; Haake, R.; Blazar, B.; Miller, W.; McGlave, P.; Ramsay, N.; Kersey, J.; Filipovich, A. Treatment of moderate/severe acute graft-versus-host disease after allogeneic bone marrow transplantation: An analysis of clinical risk features and outcome. Blood 1990, 75, 1024-1030. Available online: https:/ / pubmed.ncbi.nlm.nih.gov/2302454/ (accessed on 27 August 2020). [CrossRef] [PubMed]

5. Copelan, E.A. Hematopoietic stem-cell transplantation. N. Engl. J. Med. 2006, 354, 1813-1826. Available online: https://pubmed. ncbi.nlm.nih.gov/16641398/ (accessed on 27 August 2020). [CrossRef]

6. Bhatia, S.; Francisco, L.; Carter, A.; Sun, C.-L.; Baker, K.S.; Gurney, J.G.; McGlave, P.B.; Nademanee, A.; O’Donnell, M.; Ramsay, N.K.C.; et al. Late mortality after allogeneic hematopoietic cell transplantation and functional status of long-term survivors: Report from the Bone Marrow Transplant Survivor Study. Blood 2007, 110, 3784-3792. Available online: https: / /ashpublications.org/blood/article-pdf/110/10/3784/1290674/zh802207003784.pdf (accessed on 30 August 2020). [CrossRef]

7. Sun, C.L.; Francisco, L.; Kawashima, T.; Leisenring, W.; Robison, L.L.; Baker, K.S.; Weisdorf, D.J.; Forman, S.J.; Bhatia, S. Prevalence and predictors of chronic health conditions after hematopoietic cell transplantation: A report from the Bone Marrow Transplant Survivor Study. Blood 2010, 116, 3129-3139. Available online: https:/ / pubmed.ncbi.nlm.nih.gov/20656930/ (accessed on 30 August 2020). [CrossRef]

8. Decker, W.; McTurner-McGlade, J.; Fehir, K. Psychosocial aspects and the physiological effects of a cardiopulmonary exercise program in patients undergoing bone marrow transplantation (BMT) for acute leukemia (AL). Transplant. Proc. 1989, 21 Pt 3 , 3068-3069. Available online: https:/ / pubmed.ncbi.nlm.nih.gov/2650427/ (accessed on 27 August 2020). [CrossRef]

9. Curt, G.A.; Breitbart, W.; Cella, D.; Groopman, J.E.; Horning, S.J.; Itri, L.M.; Johnson, D.H.; Miaskowski, C.; Scherr, S.L.; Portenoy, R.K.; et al. Impact of Cancer-Related Fatigue on the Lives of Patients: New Findings From the Fatigue Coalition. Oncologist 2000, 5, 353-360. Available online: https:// pubmed.ncbi.nlm.nih.gov/11040270/ (accessed on 27 August 2020). [CrossRef]

10. Hacker, E.D.; Ferrans, C.; Verlen, E.; Ravandi, F.; Van Besien, K.; Gelms, J.; Dieterle, N. Fatigue and Physical Activity in Patients Undergoing Hematopoietic Stem Cell Transplant. Oncol. Nurs. Forum 2006, 33, 614-625. Available online: https: / / pubmed.ncbi.nlm.nih.gov/16676017/ (accessed on 27 August 2020). [CrossRef]

11. Hayes, S.; Davies, P.S.W.; Parker, T.; Bashford, J. Total energy expenditure and body composition changes following peripheral blood stem cell transplantation and participation in an exercise programme. Bone Marrow Transplant. 2003, 31, 331-338. [CrossRef] [PubMed]

12. Baumann, F.; Zimmer, P.; Finkenberg, K.; Hallek, M.; Bloch, W.; Elter, T. Influence of endurance exercise on the risk of pneumonia and Fever in leukemia and lymphoma patients undergoing high dose chemotherapy. A pilot Study. J. Sports Sci. Med. 2012, 11, 638-642. Available online: http:/ / www.ncbi.nlm.nih.gov/pubmed/24150073 (accessed on 28 August 2020).

13. Thonnard, J.L.; Penta, M. Functional assessment in physiotherapy. A literature review. Eura Medicophys. 2007, 43, 525-541. [PubMed]

14. Haig, A.J.; Jayarajan, S.; Maslowski, E.; Yamakawa, K.S.; Tinney, M.; Beier, K.P.; Juang, D.; Chan, L.; Boggess, T.; Loar, J.; et al. Development of a language-independent functional evaluation. Arch. Phys. Med. Rehabil. 2009, 90, 2074-2080. Available online: https:/ / pubmed.ncbi.nlm.nih.gov/19969171/ (accessed on 18 February 2021). [CrossRef] [PubMed]

15. Hayes, S.; Davies, P.S.W.; Parker, T.; Bashford, J.; Newman, B. Quality of life changes following peripheral blood stem cell transplantation and participation in a mixed-type, moderate-intensity, exercise program. Bone Marrow Transplant. 2004, 33, 553-558. Available online: http:/ /www.ncbi.nlm.nih.gov/pubmed/14716346 (accessed on 10 September 2019). [CrossRef]

16. Velasco Rami, J.A.; Maroto Montero, J.M. Cardiac rehabilitation. Its historical evolution and current situation. Rev. Esp. Cardiol. 1995, 48 (Suppl. 1), 1-7.

17. Winningham, M.L.; MacVicar, M.G. Exercise as a tension reduction mechanism in cancer patients. Ohio J. Sci. $1983,83,75$.

18. MacVicar, M.G.; Winningham, M.L.N.J. Effects of aerobic interval training on cancer patients functional capacity. Nurs. Res. 1989, 38, 348-351. Available online: https://pubmed.ncbi.nlm.nih.gov/2587289/ (accessed on 24 December 2021). [CrossRef]

19. Lucia, A.; Earnest, C.; Perez, M. Cancer-related fatigue: Can exercise physiology assist oncologists? Lancet Oncol. 2003, 4, 616-625. [CrossRef]

20. Campbell, K.L.; Winters-Stone, K.M.; Wiskemann, J.; May, A.M.; Schwartz, A.L.; Courneya, K.S.; Zucker, D.S.; Matthews, C.E.; Ligibel, J.A.; Gerber, L.H.; et al. Exercise Guidelines for Cancer Survivors: Consensus Statement from International Multidisciplinary Roundtable. Med. Sci. Sports Exerc. 2019, 51, 2375-2390. [CrossRef]

21. Dimeo, F.; Bertz, H.; Finke, J.; Fetscher, S.; Mertelsmann, R.; Keul, J. An aerobic exercise program for patients with haematological malignancies after bone marrow transplantation. Bone Marrow Transplant. 1996, 18, 1157-1160. Available online: http://www. ncbi.nlm.nih.gov/pubmed/8971388 (accessed on 30 August 2019). [PubMed]

22. Papasavvas, T.; Bonow, R.O.; Alhashemi, M.; Micklewright, D. Depression Symptom Severity and Cardiorespiratory Fitness in Healthy and Depressed Adults: A Systematic Review and Meta-Analysis. Sport Med. 2016, 46, 219-230. Available online: https:/ /link.springer.com/article/10.1007/s40279-015-0409-5 (accessed on 22 April 2021). [CrossRef] [PubMed]

23. Baumann, F.T.; Kraut, L.; Schüle, K.; Bloch, W.; Fauser, A.A. A controlled randomized study examining the effects of exercise therapy on patients undergoing haematopoietic stem cell transplantation. Bone Marrow Transplant. 2010, 45, 355-362. Available online: https:/ / pubmed.ncbi.nlm.nih.gov/19597418/ (accessed on 27 August 2020). [CrossRef] [PubMed] 
24. Wilk, M.; Gepfert, M.; Krzysztofik, M.; Mostowik, A.; Filip, A.; Hajduk, G.; Zajac, A. Impact of duration of eccentric movement in the one-repetition maximum test result in the bench press among women. J. Sport Sci. Med. 2020, 19, 317-322.

25. Drum, S.N.; Klika, R.J.; Carter, S.D.; Sprod, L.K.; Donath, L. A feasibility study related to inactive cancer survivors compared with non-cancer controls during aerobic exercise training. J. Sport Sci. Med. 2016, 15, 592-600. Available online: http://www.jssm.org (accessed on 24 February 2021).

26. Fiuza-Luces, C.; Simpson, R.J.; Ramírez, M.; Lucia, A.; Berger, N.A. Physical function and quality of life in patients with chronic GvHD: A summary of preclinical and clinical studies and a call for exercise intervention trials in patients. Bone Marrow Transplant. 2016, 51, 13-26. Available online: http://www.nature.com/articles/bmt2015195 (accessed on 27 August 2019). [CrossRef] [PubMed]

27. Dimeo, F.; Tilmann, M.H.M.; Bertz, H.; Kanz, L.; Mertelsmann, R.; Keul, J. Aerobic exercise in the rehabilitation of cancer patients after high dose chemotherapy and autologous peripheral stem cell transplantation. Cancer 1997, 79, 1717-1722. [CrossRef]

28. Dimeo, F.; Fetscher, S.; Lange, W.; Mertelsmann, R.; Keul, J. Effects of Aerobic Exercise on the Physical Performance and Incidence of Treatment-Related Complications After High-Dose Chemotherapy. Blood 1997, 90, 3390-3394. Available online: https:/ / pubmed.ncbi.nlm.nih.gov/9345021/ (accessed on 27 August 2020). [CrossRef]

29. Hacker, E.D.; Kim, I.; Park, C.; Peters, T. Real-time Fatigue and Free-Living Physical Activity in Hematopoietic Stem Cell Transplantation Cancer Survivors and Healthy Controls. Cancer Nurs. 2017, 40, 259-268. Available online: http:/ /journals.lww. com/00002820-201707000-00002 (accessed on 10 August 2020). [CrossRef]

30. DeFor, T.E.; Burns, L.J.; Gold, E.A.; Weisdorf, D.J. A Randomized Trial of the Effect of a Walking Regimen on the Functional Status of 100 Adult Allogeneic Donor Hematopoietic Cell Transplant Patients. Biol. Blood Marrow Transplant. 2007, 13, 948-955. Available online: https:/ /linkinghub.elsevier.com/retrieve/pii/S1083879107002480 (accessed on 12 August 2020). [CrossRef]

31. Jarden, M.; Hovgaard, D.; Boesen, E.; Quist, M.; Adamsen, L. Pilot study of a multimodal intervention: Mixed-type exercise and psychoeducation in patients undergoing allogeneic stem cell transplantation. Bone Marrow Transplant. 2007, 40, 793-800. Available online: http:/ / www.ncbi.nlm.nih.gov / pubmed/17704795 (accessed on 15 July 2019). [CrossRef]

32. Moher, D.; Liberati, A.; Tetzlaff, J.; Altman, D.G. Preferred Reporting Items for Systematic Reviews and Meta-Analyses: The PRISMA Statement. PLoS Med. 2009, 6, 6. [CrossRef]

33. Elkins, M.R.; Moseley, A.M.; Sherrington, C.; Herbert, R.D.; Maher, C.G. Growth in the Physiotherapy Evidence Database (PEDro) and use of the PEDro scale. Br. J. Sports Med. 2013, 47, 188-189. Available online: http://bjsm.bmj.com/lookup/doi/10.1136/ bjsports-2012-091804 (accessed on 25 August 2020). [CrossRef]

34. Shelton, M.L.; Lee, J.Q.; Morris, G.S.; Massey, P.R.; Kendall, D.G.; Munsell, M.F.; Anderson, K.O.; Simmonds, M.J.; Giralt, S.A. A randomized control trial of a supervised versus a self-directed exercise program for allogeneic stem cell transplant patients. Psychooncology 2009, 18, 353-359. Available online: http://doi.wiley.com/10.1002/pon.1505 (accessed on 15 August 2020). [CrossRef]

35. Barğı, G.; Güçlü, M.B.; Arıbaş, Z.; Akı, Ş.Z.; Sucak, G.T. Inspiratory muscle training in allogeneic hematopoietic stem cell transplantation recipients: A randomized controlled trial. Support Care Cancer 2016, 24, 647-659. Available online: http: / /link.springer.com/10.1007/s00520-015-2825-3 (accessed on 16 August 2020). [CrossRef]

36. Wiskemann, J.; Dreger, P.; Schwerdtfeger, R.; Bondong, A.; Huber, G.; Kleindienst, N.; Ulrich, C.M.; Bohus, M. Effects of a partly self-administered exercise program before, during, and after allogeneic stem cell transplantation. Blood 2011, 117, $2604-2613$. [CrossRef]

37. Hacker, E.D.; Larson, J.; Kujath, A.; Peace, D.; Rondelli, D.; Gaston, L. Strength Training Following Hematopoietic Stem Cell Transplantation. Cancer Nurs. 2011, 34, 238-249. Available online: http://journals.lww.com/00002820-201105000-00011 (accessed on 15 August 2020). [CrossRef]

38. Baumann, F.; Zopf, E.M.; Nykamp, E.; Kraut, L.; Schüle, K.; Elter, T.; Fauser, A.; Bloch, W. Physical activity for patients undergoing an allogeneic hematopoietic stem cell transplantation: Benefits of a moderate exercise intervention. Eur. J. Haematol. 2011, 87, 148-156. Available online: http://doi.wiley.com/10.1111/j.1600-0609.2011.01640.x (accessed on 15 August 2020). [CrossRef]

39. Coleman, E.A.; Coon, S.; Hall-Barrow, J.; Richards, K.; Gaylor, D.; Stewart, B. Feasibility of Exercise During Treatment for Multiple Myeloma. Cancer Nurs. 2003, 26, 410-419. Available online: http:/ /journals.lww.com/00002820-200310000-00012 (accessed on 16 August 2020). [CrossRef]

40. Jarden, M.; Nelausen, K.; Hovgaard, D.; Boesen, E.; Adamsen, L. The Effect of a Multimodal Intervention on Treatment-Related Symptoms in Patients Undergoing Hematopoietic Stem Cell Transplantation: A Randomized Controlled Trial. J. Pain Symptom Manag. 2009, 38, 174-190. [CrossRef]

41. Kim, S.D.; Kim, H.S. A series of bed exercises to improve lymphocyte count in allogeneic bone marrow transplantation patients. Eur. J. Cancer Care 2006, 15, 453-457. Available online: http:/ / www.ncbi.nlm.nih.gov/pubmed/17177902 (accessed on 16 August 2019). [CrossRef]

42. Jarden, M.; Baadsgaard, M.; Hovgaard, D.; Boesen, E.; Adamsen, L. A randomized trial on the effect of a multimodal intervention on physical capacity, functional performance and quality of life in adult patients undergoing allogeneic SCT. Bone Marrow Transplant. 2009, 43, 725-737. [CrossRef] 
43. Knols, R.H.; de Bruin, E.D.; Uebelhart, D.; Aufdemkampe, G.; Schanz, U.; Stenner-Liewen, F.; Hitz, F.; Taverna, C.; Aaronson, N.K. Effects of an outpatient physical exercise program on hematopoietic stem-cell transplantation recipients: A randomized clinical trial. Bone Marrow Transplant. 2011, 46, 1245-1255. Available online: http:/ / www.ncbi.nlm.nih.gov/pubmed/21132025 (accessed on 31 August 2019). [CrossRef]

44. Schumacher, H.; Stüwe, S.; Kropp, P.; Diedrich, D.; Freitag, S.; Greger, N.; Junghanss, C.; Freund, M.; Hilgendorf, I. A prospective, randomized evaluation of the feasibility of exergaming on patients undergoing hematopoietic stem cell transplantation. Bone Marrow Transplant. 2018, 53, 584-590. [CrossRef]

45. Wiskemann, J.; Kuehl, R.; Dreger, P.; Schwerdtfeger, R.; Huber, G.; Ulrich, C.M.; Jaeger, D.; Bohus, M. Efficacy of exercise training in SCT patients-who benefits most? Bone Marrow Transplant. 2014, 49, 443-448. [CrossRef]

46. Mello, M.; Tanaka, C.; Dulley, F.L. Effects of an exercise program on muscle performance in patients undergoing allogeneic bone marrow transplantation. Bone Marrow Transplant. 2003, 32, 723-728. Available online: http://www.ncbi.nlm.nih.gov/pubmed/13 130321 (accessed on 31 August 2019). [CrossRef]

47. Wiskemann, J.; Kleindienst, N.; Kuehl, R.; Dreger, P.; Schwerdtfeger, R.; Bohus, M. Effects of physical exercise on survival after allogeneic stem cell transplantation. Int. J. Cancer. 2015, 137, 2749-2756. Available online: http://doi.wiley.com/10.1002/ijc.29633 (accessed on 17 August 2020). [CrossRef]

48. Peters, T.; Erdmann, R.; Hacker, E.D. Exercise intervention: Attrition, compliance, adherence, and progression following hematopoietic stem cell transplantation. Clin. J. Oncol. Nurs. 2018, 22, 97-103. [CrossRef] [PubMed]

49. Pahl, A.; Wehrle, A.; Kneis, S.; Gollhofer, A.; Bertz, H. Feasibility of whole body vibration during intensive chemotherapy in patients with hematological malignancies-A randomized controlled pilot study. BMC Cancer 2018, 18, 920. Available online: https:/ / bmccancer.biomedcentral.com/articles/10.1186/s12885-018-4813-8 (accessed on 19 November 2020). [CrossRef]

50. Persoon, S.; ChinAPaw, M.; Buffart, L.; Liu, R.K.; Wijermans, P.; Koene, H.; Minnema, M.; Lugtenburg, P.; Marijt, E.W.A.; Brug, J.; et al. Randomized controlled trial on the effects of a supervised high intensity exercise program in patients with a hematologic malignancy treated with autologous stem cell transplantation: Results from the EXIST study. PLoS ONE 2017, 12, 15. Available online: http:/ / www.ncbi.nlm.nih.gov/pubmed/28727771 (accessed on 18 August 2020). [CrossRef]

51. Bird, L.; Arthur, A.; Niblock, T.; Stone, R.; Watson, L.; Cox, K. Rehabilitation programme after stem cell transplantation: Randomized controlled trial. J. Adv. Nurs. 2010, 66, 607-615. Available online: http://doi.wiley.com/10.1111/j.1365-2648.2009.0 5232.x (accessed on 16 August 2020). [CrossRef] [PubMed]

52. Oechsle, K.; Aslan, Z.; Suesse, Y.; Jensen, W.; Bokemeyer, C.; de Wit, M. Multimodal exercise training during myeloablative chemotherapy: A prospective randomized pilot trial. Support Care Cancer 2014, 22, 63-69. Available online: http:/ / link.springer. com/10.1007/s00520-013-1927-z (accessed on 18 November 2020). [CrossRef] [PubMed]

53. Van Dongen, J.M.; Persoon, S.; Jongeneel, G.; Bosmans, J.E.; Kersten, M.J.; Brug, J.; Nollet, F.; Chinapaw, M.J.M.; Buffart, L.M. Long-term effectiveness and cost-effectiveness of an 18-week supervised exercise program in patients treated with autologous stem cell transplantation: Results from the EXIST study. J. Cancer Surviv. 2019, 13, 558-569. Available online: http:/ /link.springer. com/10.1007/s11764-019-00775-9 (accessed on 18 November 2020). [CrossRef] [PubMed]

54. Pahl, A.; Wehrle, A.; Kneis, S.; Gollhofer, A.; Bertz, H. Whole body vibration training during allogeneic hematopoietic cell transplantation-The effects on patients' physical capacity. Ann. Hematol. 2020, 99, 635-648. [CrossRef]

55. De Almeida, L.B.; Trevizan, P.F.; Laterza, M.C.; Neto, A.E.H.; de São José, A.C.A.; Martinez, D.G. Safety and feasibility of inspiratory muscle training for hospitalized patients undergoing hematopoietic stem cell transplantation: A randomized controlled study. Support Care Cancer. 2020, 28, 3627-3635. [CrossRef]

56. Van Haren, I.E.P.M.; Timmerman, H.; Potting, C.M.; Blijlevens, N.M.A.; Staal, J.B.; Nijhuis-van der Sanden, M.W.G. Physical exercise for patients undergoing hematopoietic stem cell transplantation: Systematic review and meta-analyses of randomized controlled trials. Phys. Ther. 2013, 93, 514-528. Available online: https:/ / pubmed.ncbi.nlm.nih.gov/23224217/ (accessed on 21 July 2021). [CrossRef]

57. Persoon, S.; Kersten, M.J.; van der Weiden, K.; Buffart, L.M.; Nollet, F.; Brug, J.; Chinapaw, M.J.M. Effects of exercise in patients treated with stem cell transplantation for a hematologic malignancy: A systematic review and meta-analysis. Cancer Treat. Rev. 2013, 39, 682-690. [CrossRef]

58. Issues, S.; Test, M.W.; Equipment, R.; Preparation, P. American Thoracic Society ATS Statement: Guidelines for the Six-Minute Walk Test. Am. J. Respir. Crit. Care Med. 2002, 166, 111-117.

59. Convertino, V.A. Cardiovascular consequences of bed rest: Effect on maximal oxygen uptake. Med. Sci. Sports Exerc. 1997, 29, 191-196. Available online: https:/ / pubmed.ncbi.nlm.nih.gov/9044222/ (accessed on 29 May 2021). [CrossRef]

60. Al-Majid, S.; Mccarthy, D.O. Cancer-Induced Fatigue and Skeletal Muscle Wasting: The Role of Exercise. Biol. Res. Nurs. 2001, 2, 186-197. Available online: https:/ / pubmed.ncbi.nlm.nih.gov/11547540/ (accessed on 2 July 2021). [CrossRef]

61. Morishita, S.; Kaida, K.; Yamauchi, S.; Wakasugi, T.; Ikegame, K.; Ogawa, H.; Domen, K. Relationship of physical activity with physical function and health-related quality of life in patients having undergone allogeneic haematopoietic stem-cell transplantation. Eur. J. Cancer Care 2017, 26, e12669. [CrossRef] [PubMed]

62. Courneya, K.; Friedenreich, C.M. Physical exercise and quality of life following cancer diagnosis: A literature review. Ann. Behav. Med. 1999, 21, 171-179. Available online: https:/ /academic.oup.com/abm/article/21/2/171-179/4631432 (accessed on 27 August 2020). [CrossRef] [PubMed] 
63. Van Haren, I.E.P.M.; Staal, J.B.; Potting, C.M.; Atsma, F.; Hoogeboom, T.J.; Blijlevens, N.M.A.; Nijhuis-van der Sanden, M.W.G. Physical exercise prior to hematopoietic stem cell transplantation: A feasibility study. Physiother. Theory Pract. 2018, 34, 747-756. [CrossRef] [PubMed]

64. Hacker, E.D.; Mjukian, M. Review of attrition and adherence in exercise studies following hematopoietic stem cell transplantation. Eur. J. Oncol. Nurs. 2014, 18, 175-182. [CrossRef] [PubMed] 